\title{
A Robust Analysis of the Risk-Structure of Equilibrium Term Structures of Bond Yields
}

\author{
Anh Le and Kenneth J. Singleton*
}

August 15, 2012

\begin{abstract}
Many equilibrium term structure models $(E T S M \mathrm{~s})$ in which the state of the economy $z_{t}$ follows an affine process imply that the variation in expected excess returns on bond portfolio positions is fully spanned by the set of conditional variances $\varsigma_{t}^{2}$ of $z_{t}$. We show that these two assumptions alone- spanning of expected excess returns by the variances $\varsigma_{t}^{2}$ of affine processes $z_{t}$ - are sufficient to econometrically identify the quantities of risk that span risk premiums from the term structure of bond yields. Using this result we derive maximum likelihood estimates of $\varsigma_{t}^{2}$ and evaluate the goodness-of-fit of the family of affine ETSMs that imply this tight link between premiums and quantities of risk. These assessments are fully robust to the values of the parameters governing preferences and the evolution of the state $z_{t}$, and to whether or not the economy is arbitrage free. Our findings suggest that, to be consistent with U.S. macroeconomic and Treasury yield data, affine ETSMs should have the features that: (i) the fundamental sources of risks, including consumption growth, inflation, and yield volatilities are driven by distinct economic shocks; (ii) consumption growth risk alone does not fully account for the predictability of excess returns on bonds; and (iii) inflation risk, and not long-run risks or variation in risk premiums arising from habit-based preferences, is a significant (and perhaps the dominant) risk underlying risk premiums in U.S. Treasury markets.
\end{abstract}

${ }^{*}$ Le: Kenan-Flagler Business School, University of North Carolina at Chapel Hill, Chapel Hill, NC 27599 (email: anh_le@unc.edu); Singleton: Graduate School of Business, Stanford University, Stanford, CA 94305, and National Bureau of Economic Research (email: kenneths@stanford.edu). 


\section{Introduction}

Equilibrium dynamic term structure models $(E T S M \mathrm{~s})$ that specify agents' marginal rate of substitution in an exponential-affine form ${ }^{1}$ imply that expected excess returns from holding traded securities are fully determined by the underlying sources of time-varying volatilities in the state variables, regardless of the source of variation in risk premiums. In models with habit formation (e.g., Campbell and Cochrane (1999), Wachter (2006)) it is the time-varying volatility of the surplus consumption ratio that induces time-variation in the market price of consumption risk, while the quantity of consumption risk is held constant. Alternatively, in the LRR models of Bansal, Kiku, and Yaron (2007) and Bansal and Shaliastovich (2010), the market prices of consumption risks are constant and variation in excess returns is fully explained by variation in the quantities of these risks.

This paper explores in depth the nature of risk premiums in US Treasury bond markets over the past thirty years through the lens of investors' pricing kernels as parameterized in these (and other) studies of preference-based ETSMs. Bonds are a natural asset class for exploring the empirical relevance of consumption risks because the availability of a broad spectrum of maturities provides a market-based parsing of the effects of short- and long-lived risk factors on excess returns. Moreover for default-free debt, and absent strong clientele effects along the yield curve, ${ }^{2}$ yields on bonds of all maturities depend on the same underlying risk factors and, as such, there is a rich cross-section of information about the economic risks underlying the risk premiums demanded by market participants. Bond yields highlight variation in discount rates (as opposed to the cash-flow risks in equity markets) where, arguably, the contributions of consumption risks are most clearly revealed.

Our approach blends the structure of the consumption risks embodied in typical models with habit formation or $L R R$ with the focus on factor structures and market prices of risk in reduced-form, affine term structure models (Dai and Singleton (2003), Piazzesi (2010)). We highlight two robust features of recent ETSMs: First, they imply that the only sources of variation in expected excess returns on bonds are the time-varying volatilities of the risk factors underlying consumption growth and $L R R$ or surplus consumption. More concretely, their assumptions about agents' pricing kernel, the conditional distributions of the risk factors, and the market prices of these factor risks give rise to factor representations of expected excess returns on bonds with known dimension, and the factors driving risk premiums are a subset of those determining the cross-sectional distribution of bond yields. Second ETSMs, as typically formulated, imply full spanning of expected consumption growth and expected inflation by bond yields. Combining these observations, it follows that information about the growth component of aggregate consumption is irrelevant for modeling risk premiums on bonds after accounting for the forecasting power of risk-factor volatilities.

\footnotetext{
${ }^{1}$ See, for instance, Eraker and Shaliastovich (2008) and Eraker (2008) for discussions of equilibrium affine pricing models based on recursive preferences and long-run risks (LRR), and Le, Singleton, and Dai (2010) for a nonlinear model with habit formation that admits affine pricing.

${ }^{2}$ Such segmentation effects have been recently explored by Greenwood and Vayanos (2010a), Krishnamurthy and Vissing-Jorgensen (2010), and Vayanos and Vila (2009), among others. As in most of the empirical research on risk premiums in bond markets, we abstract from supply effects.
} 
A premise of most of our econometric analysis is that equilibrium bond yields are affine functions of their underlying risk factors. Most ETSMs with $L R R$ are constructed so that yields follow an $N$-factor affine model, with $N$ typically ranging between two and five. For instance, the LRR model of explored by Bansal and Shaliastovich (2010) (hereafter B-S) and Koijen et al. (2010) implies a four-factor ETSM, two more than its predecessor in Bansal and Yaron (2004). The habit-based ETSMs of Wachter (2006) and Le et al. (2010) give rise to two-factor models. With these models in mind, we proceed to extract up to four risk factors from time-series data on a broad cross-section of yields. ${ }^{3}$

That the risk factors in ETSMs can be extracted from market returns has long figured prominently in the literature on pricing equity portfolios. For instance, Bansal, Kiku, and Yaron (2007), Constantinides and Ghosh (2009), and Marakani (2009) explore the fits of two-factor models with $L R R$ using the price-dividend ratio for the aggregate stock market and the nominal risk-free rate to extract a $L R R$ factor from asset returns and consumption data. Our complementary analysis of bond returns differs in three important respects, all linked to the fact that bond yields depend only on the fundamental risk factors underlying the structure of $L R R$ (consumption growth) and inflation, and not on individual cash-flow risks. First, we focus on a four-factor model of $L R R$, thereby accommodating the richer consumption and inflation dynamics in B-S's model. Second, the availability of yields on bonds with a cross-section of maturities allows us to evaluate the model-implied factor structure of risk premiums without having to use consumption data. We therefore avoid any measurement issues associated with consumption, and we are able to evaluate key implications of $L R R$ models using well-measured monthly yield data. ${ }^{4}$ Third, we exploit links between the factor structures of expected excess returns on bonds and of consumption growth to evaluate implied constraints on the $L R R$ factor itself.

Central to our empirical analysis of ETSMs is a new set of data on yields on US Treasury zero-coupon bonds constructed from daily data on a large cross-section of yields. The FamaBliss CRSP and Gurkanyak, Sack, and Wright (2007) (GSW) datasets are the most widely used for empirical analysis of dynamic term structure models (DTSMs). The former only contains maturities out to five years (a limitation in our view for studying risk premiums in equilibrium models), while a limitation of the latter is that the authors construct smoothed fitted yields using an extended Nelson and Siegel (1987) model. Using the extensive CRSP database on yields on individual Treasury coupon bonds, and applying the same filter to remove bonds that are illiquid or have embedded options, and the same Fama-Bliss bootstrap method as CRSP (see Bliss (1997)), we construct a consistent set of zero-coupon bond yields with maturities out to ten years over a sample period from 1972 through 2007. As we document subsequently, there is substantial predictive power of long-dated forward rates for excess returns relative to what is found with the smoothed GSW data. This feature of our data will play a key role in the subsequent assessment of the nature of the economic forces

\footnotetext{
${ }^{3}$ Empirical implementations of reduced-form affine term structure models have typically found that three to four factors explain both the cross-section and time-series properties of bond yields.

${ }^{4}$ Much of the debate about the goodness-of-fit of $L R R$ models to macroeconomic data has focused on measurement issues associated with consumption. See, for instance, the discussions in Bansal, Kiku, and Yaron (2007) and Beeler and Campbell (2009).
} 


\begin{tabular}{|lcccccc|}
\hline Recursive Preferences & LRR & $N$ & Priced Risks & $\mathcal{R}$ & RP & MPR \\
Bansal, Kiku, and Yaron (2007) & yes & 3 & $x_{t}, \sigma_{g t}^{2}, \bar{\pi}_{t}$ & 1 & $\sigma_{g t}^{2}$ & const \\
Bansal and Shaliastovich (2010) & yes & 4 & $x_{t}, \sigma_{g t}^{2}, \sigma_{x t}^{2}, \bar{\pi}_{t}$ & 2 & $\sigma_{g t}^{2}, \sigma_{x t}^{2}$ & const \\
Bollerslev, Tauchen, and Zhou (2009) & no & 3 & $\sigma_{g t}^{2}, q_{t}, \bar{\pi}_{t}$ & 2 & $\sigma_{g t}^{2}, q_{t}$ & const \\
Drechsler and Yaron (2011) & yes & 6 & $x_{t}, \sigma_{g t}^{2}, q_{t}, \pi_{t}$ & 1 & $\sigma_{g t}^{2}$ & const \\
& & & Jump Risks & & & \\
\hline Habit Formation & LRR & $N$ & Priced Risks & $\mathcal{R}$ & RP & MPR \\
Wachter (2006) & no & 2 & $s_{t}, \bar{\pi}_{t}$ & 1 & $s_{t}$ & t-vary \\
Le, Singleton, and Dai (2010) & no & 2 & $s_{t}, \bar{\pi}_{t}$ & 1 & $s_{t}$ & t-vary \\
\hline
\end{tabular}

Table 1: Features of ETSMs. The column "LRR" indicates whether the model has $L R R$; $N$ is the number of priced risks which are indicated in column four; $\mathcal{R}$ is the model-implied dimension of expected excess returns on nominal bonds, and the factors driving these risk premiums are indicated in column six; and the column "MPR" indicates whether the market prices of risk are constant or time-varying (t-vary).

underlying risk premiums.

\section{The Factor Structure of ETSMs}

Consider an endowment economy, as in most of the extant literature, and let $C_{t}$ denote real per-capita consumption and $c_{t} \equiv \log C_{t}$. We focus on a consumption growth process that encompasses those examined in both the $L R R$ and habit literatures:

$$
\begin{aligned}
\Delta c_{t+1} & =\mu_{g}+x_{t}+\eta_{t+1} \\
x_{t+1} & =\rho x_{t}+e_{t+1}
\end{aligned}
$$

where $x_{t}$ is the $L R R$ factor and the consumption-growth and $L R R$ shocks $\left(\eta_{t+1}, e_{t+1}\right)$ follow affine distributions, conditional on agents' date $t$ information. This specification nests the consumption processes in the models of Bansal, Kiku, and Yaron (2007), Bansal and Shaliastovich (2010), Bollerslev, Tauchen, and Zhou (2009), and Drechsler and Yaron (2011); as well as the habit-based models of Wachter (2006) and Le, Singleton, and Dai (2010), and preference shock model of Bekaert and Engstrom (2010). The conditional variances of the shocks $\left(\eta_{t+1}, e_{t+1}\right)$ may have a multi-dimensional factor structure, as for instance in Bollerslev, Tauchen, and Zhou (2009) and Bekaert and Engstrom (2010). These shocks may also embody jump components with state-dependent arrival intensities as in Drechsler and Yaron (2011). All of these models are encompassed by our assumption that the shocks follow affine processes (e.g., Duffie, Pan, and Singleton (2000)). Table 1 summarizes the features of these models that are particularly relevant to the goals of our analysis. 
The logarithm of the kernel for pricing nominal bonds typically takes the form

$$
m_{t+1}=\gamma_{0} \log \delta-\gamma_{1} \Delta c_{t+1}-\gamma_{2} \varphi_{t+1}-\pi_{t+1}
$$

where $\pi_{t+1}=\log \left(P_{t+1} / P_{t}\right)$, with $P_{t}$ denoting the price level, and $\bar{\pi}_{t}$ is the conditional mean of one-period-ahead inflation. ${ }^{5}$ In the models of habit formation $\varphi_{t+1}$ is the growth rate of the consumption surplus ratio. Letting $H_{t}$ denote agents' level of external habit, $s_{t}=\log \left[\left(C_{t}-H_{t}\right) / C_{t}\right]$ and $\varphi_{t+1}=\left(s_{t+1}-s_{t}\right)$. Wachter (2006) and Le et al. (2010) examine models with two priced risks $(N=2), s_{t}$ and $\pi_{t}$. Consumption growth is assumed to be conditionally perfectly correlated with $s_{t}$, as in Campbell and Cochrane (1999). Bekaert and Engstrom (2010) assume $C R R A$ preferences over the consumption process (1)-(2) which gives the special case of (3) in which $\varphi_{t}$ is an exogenous preference shock, and the conditional variances of $\left(\eta_{t}, e_{t}\right)$ differ in "good" and "bad" economic times.

In the model with $L R R$ explored by Bansal and Yaron (2004) and Bansal, Kiku, and Yaron (2007), among others, $m_{t+1}$ is given by (3) with $\varphi_{t+1}=r_{c, t+1}$, the one-period return on a claim to aggregate consumption flows. These studies have three priced risks under the assumption that the conditional variances of $\eta_{t+1}$ and $e_{t+1}$ are the same $\left(\sigma_{g t}^{2}\right)$, while Bansal and Shaliastovich (2010) assume these shocks have different conditional variance processes $(N=4)$. The largest number of priced risks arises in the model of Drechsler and Yaron (2011) where, to the priced risks in Bansal et al. (2007), they add a stochastic drift to $\sigma_{g t}^{2}$ (denoted $q_{t}$ in Table 1 ) and jumps in both $\sigma_{g t}^{2}$ and $x_{t}$. Their jump intensities are assumed to be affine functions of $\sigma_{g t}^{2}$, thereby ensuring that their state follows an affine process. Finally, Bollerslev et al. (2009) allow the conditional variance of $\sigma_{g t}^{2}\left(q_{t}\right)$ to be time-varying and to exhibit stochastic volatility. This gives rise to an additional priced volatility risk.

Common to all of these equilibrium models is the feature that the dimensions $(\mathcal{R})$ of the expected excess returns or risk premiums $(R P)$ are less than the dimensions of the risk factors underlying bond yields $(N>\mathcal{R})$. In the models with $L R R$, time variation in expected excess returns is induced entirely by variation in the consumption and $L R R$ volatilities that follow first-order Markov processes. Drechsler and Yaron (2011) have the largest number of priced risks underlying bond yields, but the structure of their model is such that expected excess returns on all bonds of all maturities are proportional to $\sigma_{g t}^{2}($ so $\mathcal{R}=1$ ).

The habit-based models of Wachter (2006) and Le et al. (2010) share the common feature, following Campbell and Cochrane (1999), that innovations in consumption growth and surplus consumption are perfectly correlated and have a time-varying volatility induced by variation in surplus consumption. As such, expected excess returns in these models also lie in a one-dimensional space determined by $s_{t}$. On the other hand, Bekaert and Engstrom (2010) allow for two affine sources of time-varying volatility in consumption growth and this implies that risk premiums in their model lie a two-dimensional linear space.

As these models illustrate, the dimensionality $\mathcal{R}$ of expected excess returns in ETSMs is determined by the number of risk factors that drive time-varying volatility. Expanding the

\footnotetext{
${ }^{5}$ Bollerslev, Tauchen, and Zhou (2009) and Drechsler and Yaron (2011) do not specify an inflation process. We proceed under the assumption that, had they been exploring the fit of their model to nominal bond yields, they would have adopted one similar to the model of Bansal and Shaliastovich (2010).
} 
number of such risks will add "dimension" (and thereby flexibility) to risk premiums. Yet, since these models have been put forth as successful representations of observed variation in expected excess returns, we proceed to evaluate their goodness-of-fit taking as given the assumed specifications of priced risks and the implied structure of risk premiums. Moreover, as we next discuss, both families of equilibrium models imply restrictions on risk premiums that are robust in the sense that they do not depend on specific values of the parameters governing preferences or on (most features of) the distributions of the state.

The expected excess return over a horizon $h$ on a $\tau$-period zero-coupon bond with yield to maturity $y_{t}^{\tau}$ is

$$
e r_{t}^{h}(\tau)=-(\tau-h) E_{t}\left[y_{t+h}^{\tau-h}\right]+\tau y_{t}^{\tau}-y_{t}^{h} .
$$

Leting $z_{t}$ denote the $N$-dimensional set of time-varying priced risks in an ETSM, all but one of the ETSMs summarized in Table 1 imply that $y_{t}^{\tau}$ is an affine function of $z_{t}$ :

$$
y_{t}^{\tau}=A(\tau)+B(\tau) \cdot z_{t}, \quad \forall \tau \geq 0 \text {. }
$$

The exception is Wachter's model which does not admit affine pricing but, to a good approximation, Le et al. (2010)'s affine pricing model nests her model (see below). Accordingly, we take (5) as given in our subsequent analysis. Then, together, (4) and (5) imply that $\operatorname{er}_{t}^{h}(\tau)$ is an affine function of $z_{t}$.

The set of pricing factors $z_{t}$ includes the expected growth rate of consumption $\overline{\Delta c}_{t}=$ $E_{t}\left[\Delta c_{t+1}\right]$ (the $L R R$ factor in those models with this feature) and expected inflation $\bar{\pi}_{t}=$ $E_{t}\left[\pi_{t+1}\right]$. It also includes the $\mathcal{R}$ volatility factors $\varsigma_{t}^{2}$ that gives rise to time-varying volatility in bond yields. In models with Epstein-Zin preferences and $L R R$, these are the conditional variances of consumption growth $\left(\sigma_{g t}^{2}\right)$ and $x_{t}=\overline{\Delta c}_{t}\left(\sigma_{x t}^{2}\right)$. In the habit-based models $\varsigma_{t}^{2}$ is the scalar surplus consumption $s_{t}$.

A particular focus of our analysis is the stronger implication of these ETSMs that expected excess returns can be expressed as

$$
e r_{t}^{h}(\tau)=\mathcal{A}(\tau, h)+\mathcal{B}(\tau, h) \cdot \varsigma_{t}^{2}
$$

where $\varsigma_{t}^{2}$ is a strict subset of the state $z_{t}$ comprised of the $\mathcal{R}$ volatility factors. These observations lead us to the following robust implication of these ETSMs:

RIETSM: The dimensionality $\mathcal{R}$ of the expected excess returns $\mathrm{er}_{t}^{h}(\tau)$ is common for all horizons $h$ and all bond maturities $\tau$. Moreover, the state vector $\varsigma_{t}^{2}$ underlying variation in the $\operatorname{er}_{t}^{h}(\tau)$ is a subset of the state vector $z_{t}$ determining bond yields, and they are the same factors which give rise to time-varying volatility in bond yields. That is, the $\varsigma_{t}^{2}$ listed under "RP" in Table 1 span the time-varying volatilities of bond yields.

At the heart of RIETSM is the assumption in all of the ETSMs except those with habitbased preferences that the market prices of risk $\Lambda$ for the risk factors $z_{t}$ are state-independent (the weights on $t+1$ variables in (3) are constants). Evidence that information other than $\varsigma_{t}^{2}$ is incrementally useful for forecasting excess returns indicates either that these ETSMs have omitted time-varying quantities of risks that are relevant in bond markets, or that the 
market prices $\Lambda_{t}$ of the risks $\varsigma_{t}^{2}$ are in fact time varying. Holding $\mathcal{R}$ fixed, state-dependence of the market prices of risk could arise either because the linearizations inherent in ETSMs that give rise to their affine structure leave out empirically relevant features of risk in bond markets, ${ }^{6}$ or because of a more fundamental mis-specification of the structure of preferences of bond investors. ${ }^{7}$

As for habit-based models, Le et al. (2010) explore a model in which $m_{t+1}$ has a non-affine structure in order to simultaneously ensure that that zero-coupon yields are exponential-affine functions of $z$ and that their model (approximately) nests prior habit-based term structure models. The expected excess returns $e r_{t}^{1}(\tau)$, for all $\tau$, are approximately affine functions of $s_{t}$ and $\sqrt{s_{t}}$ (where $s_{t}$ is surplus consumption). The precision of (6), now viewed as a linearization of this implied nonlinear interplay between the time-varying quantity and market price of $s_{t}$ risk, will depend on the specific formulation and the parameter values. Evaluated at their maximum likelihood estimates, virtually all of the variation in $e r_{t}^{1}(\tau)$ is induced by $s_{t}$, so the affine approximation is very accurate. We henceforth proceed under the assumption that (6) is a reliable approximation for all of the ETSMs considered.

\section{Robust Evaluation of the Constraint that Expected Excess Returns are Spanned by Volatility Factors}

RIETSM is a powerful implication of ETSMs. When combined with the assumption that $z_{t}$ follows an affine process, it allows us to extract a set of $\mathcal{R}$ risk factors from the term structure of bond yields that fully span expected excess returns, even without presuming that bond markets are arbitrage free. Moreover, it leads to tests of goodness-of-fit of affine ETSMs that are robust to values of the parameters governing agents preferences and the distributions of the non-volatility factors impacting bond yields.

Suppose that, consistent with the affine structure of the families of ETSMs being examined, the n-period yield $y_{n, t}$ follows the linear $N$-factor structure

$$
y_{n, t}=\left(A_{n}+B_{n} x_{t}+C_{n} \varsigma_{t}^{2}\right) / n,
$$

where the $M$ factors $\varsigma_{t}^{2}$ determine the time-varying volatility of the entire set of $N$ factors $z_{t}^{\prime}=\left(x_{t}^{\prime}, \varsigma_{t}^{2 \prime}\right)$. Additionally, the conditional means of $\varsigma_{t+1}^{2}$ and $x_{t+1}$ have the standard affine structure (ignoring constants):

$$
E_{t}\left[\varsigma_{t+1}^{2}\right]=\rho \varsigma_{t}^{2}, \quad \text { and } \quad E_{t}\left[x_{t+1}\right]=K_{1 \varsigma} \varsigma_{t}^{2}+K_{1 x} x_{t} .
$$

Importantly, to maintain admissibility, the conditional means of $\varsigma_{t+1}^{2}$ cannot be dependent on the non-volatility factor $x_{t}$ as its support is typically $\mathbb{R}^{N-M}$.

\footnotetext{
${ }^{6}$ Bansal and Yaron (2004) and Bansal and Shaliastovich (2010), among others, argue that the linearizations within their $L R R$ models are inconsequential for their empirical analyses.

${ }^{7}$ Bonomo et al. (2010), for instance, argue that replacing Kreps-Porteous preferences by preferences exhibiting disappointment aversion resolves some of the weaknesses of the Bansal and Yaron (2004) model with regard to matching the predictive power of dividend yields for consumption growth and excess returns on stocks. The pricing kernel implied by their model implicitly exhibits time-varying market prices of $L R R$.
} 
Substituting the factor representation (7), and the conditional means in (8) into (4), the one-period expected excess return for an n-period bond is, ignoring constants,

$$
\operatorname{er}_{t}^{1}(n)=\left(B_{n}-B_{n-1} K_{1 x}-B_{1}\right) x_{t}+\left(C_{n}-C_{n-1} \rho-B_{n-1} K_{1 \varsigma}-C_{1}\right) \varsigma_{t}^{2} .
$$

Under RIETSM- only $\varsigma_{t}^{2}$ predicts excess returns- the loadings $B_{n}$ must satisfy the recursion

$$
B_{n}=B_{n-1} K_{1 x}+B_{1}
$$

in which case (9) simplifies to

$$
e r_{t}^{1}(n)=\left(C_{n}-C_{n-1} \rho-B_{n-1} K_{1 \varsigma}-C_{1}\right) \varsigma_{t}^{2}
$$

Notably, though the recursion in (10) resembles those found in standard affine pricing models under the risk-neutral measure, these expressions are determined entirely by the historical $(\mathbb{P})$ distribution of $z_{t}$. This structure on $e r_{t}^{1}(n)$ is a direct implication of the complete spanning of expected excess returns by $\varsigma_{t}^{2}$ and, in particular, it does not require (or imply) arbitrage-free pricing nor an explicit volatility specification. It is worth emphasizing that even if volatility is completely unspanned by yields- $C_{n} \equiv 0$ for all n's- equations (10) and (11) still must hold.

To help with intuition, suppose there is a pricing measure $\tilde{\mathbb{Q}}$ (not necessarily equivalent to the risk-neutral measure since we do not enforce no-arbitrage) that gives rise to the pricing relation in (10). Then $K_{1 x}$ governs the feedback of $x_{t}$ under both the $\mathbb{P}$ and $\tilde{\mathbb{Q}}$ measures. By analogy to standard risk-neutral pricing, the identical feedback matrices under $\mathbb{P}$ and $\tilde{\mathbb{Q}}$ imply that $x_{t}$ does not impact risk premiums. Moreover, (10) implies that $B_{n}=B_{n-h} K_{1 x}^{h}+B_{h}$ for any horizon $h$ and, therefore, from (9) it follows that the $h$-period expected excess return of an $n$-period bond $\operatorname{er}_{t}^{h}(n)$ depends only on $\varsigma_{t}^{2}$. In other words, the recursion in (10) guarantees that $\varsigma_{t}^{2}$ completely spans expected excess returns for any horizon.

We next show how this structure can be conveniently used to obtain very strong identification of the volatility factors $\varsigma_{t}^{2}$. Throughout we assume that model assessment is based on the collection of $J$ yields $y_{t}(J>N)$ where: ${ }^{8}$

$$
y_{t}=A+B x_{t}+C \varsigma_{t}^{2},
$$

and that there exists an $N \times J$ full-rank weight matrix $W$ such that the $N$ portfolios of yields $\mathcal{P}_{t}=W y_{t}$ are measured without errors. This is analogous to the assumption in arbitrage-free dynamic term structure models that $N$ portfolios of yields are priced perfectly by the model. As documented in Joslin, Le, and Singleton (2012), when $W$ is chosen to be the loadings on the first $N$ principal components $(P C \mathrm{~s})$ of yields and $N$ is not too large, then quantification of the risk profile of bonds is robust to this exact pricing assumption.

For the time being we follow the extant literature on ETSMs (as summarized in Table 1) and assume that $\varsigma^{2}$ is fully spanned by bond yields. Volatility spanning is a strong assumption that implies that expected excess returns on all bonds over all holding periods are fully

\footnotetext{
${ }^{8}$ Specifically, let denote the maturities used in assessment by $m_{1}, m_{2}, \ldots, m_{J}$ then $A=$ $\left(A_{m_{1}} / m_{1}, A_{m_{2}} / m_{2}, \ldots, A_{m_{J}} / m_{J}\right)^{\prime} . B$ and $C$ are constructed similarly.
} 
spanned by either the yield portfolios $\mathcal{P}_{t}$ or $\varsigma_{t}^{2}$. Economic motivations for relaxing this spanning assumption, and their implications for time-varying risk premiums in bond markets, are explored in Section 6.

To express $\varsigma_{t}^{2}$ in terms of a subset of the yield portfolios $\mathcal{P}_{t}$, we partition the loading matrix $W$ into $W_{x}(N-M \times J)$ and $W_{\varsigma}(M \times J)$ and let $\mathcal{P}_{x t}=W_{x} y_{t}$ and $\mathcal{P}_{\varsigma t}=W_{\varsigma} y_{t}$. Multiplying both sides of (12) by $W_{x}$ and inverting for $x_{t}$ gives

$$
x_{t}=\left(W_{x} B\right)^{-1}\left(\mathcal{P}_{x t}-W_{x}\left(A+C \varsigma_{t}^{2}\right)\right) .
$$

Substituting back into (12) leads to an expression for $y_{t}$ in terms of $\mathcal{P}_{x t}$ and $\varsigma_{t}^{2}$ :

$$
y_{t}=B_{\mathcal{P}} \mathcal{P}_{x t}+\phi_{x}\left(A+C \varsigma_{t}^{2}\right),
$$

where $B_{\mathcal{P}}=B\left(W_{x} B\right)^{-1}$ and $\phi_{x}=I_{J}-B_{\mathcal{P}} W_{x}$. Finally, premultiplying both sides of (14) by $W_{\varsigma}$ and solving for $\varsigma_{t}^{2}$ gives

$$
\varsigma_{t}^{2}=\left(W_{\varsigma} \phi_{x} C\right)^{-1}\left(\mathcal{P}_{\varsigma t}-W_{\varsigma} B_{\mathcal{P}} \mathcal{P}_{x t}-W_{\varsigma} \phi_{x} A\right) .
$$

The full-volatility-spanning assumption guarantees that the leading matrix $W_{\varsigma} \phi_{x} C$ in (15) is invertible. It follows that, up to an affine transformation, $\varsigma_{t}^{2}$ is determined by $\mathcal{P}_{\varsigma t}-W_{\varsigma} B_{\mathcal{P}} \mathcal{P}_{x t}$.

The yield portfolios $\mathcal{P}_{s t}$ and $\mathcal{P}_{x t}$ are pre-specified linear combinations of $y_{t}$. Moreover, $B_{\mathcal{P}}$ is fully determined by (the Jordan form of) $K_{1 x}$. The latter follows from the observation that we can rotate $x$ so that $B_{1}$ is a vector of ones and $K_{1 x}$ has the Jordan form. Whence $B$ and hence $B_{\mathcal{P}}$ are completely determined by the eigenvalues of $K_{1 x}$. In the context of risk-neutral pricing, these eigenvalues are the persistence parameters $\lambda^{\mathbb{Q}}$ that Joslin, Singleton, and Zhu (2011) show are estimable with considerable precision from the cross-section of bond yields. Similarly, we anticipate (and subsequently confirm) that our identification strategy will reveal the time variation in and predictive content of $\varsigma_{t}^{2}$ very precisely through $\mathcal{P}_{\varsigma t}-W_{\varsigma} B_{\mathcal{P}} \mathcal{P}_{x t}$.

Up to this point our derivations do not exploit the fact that $\varsigma^{2}$ is a volatility process, beyond the autonomous structure of its conditional mean (8). To proceed with estimation and, most importantly, to ensure that $\varsigma_{t}^{2}$ is interpretable as the conditional volatility of $z_{t}$, we adopt a parametric affine model for the conditional distribution of $z$. Specifically, we assume that $\varsigma^{2}$ follows a multivariate autoregressive gamma $(A R G)$ process (Gourieroux and Jasiak (2006), Le, Singleton, and Dai (2010)), and the remaining $N-M$ factors $x_{t}$ are Gaussian conditional on $\varsigma_{t}^{2}$ :

$$
\begin{aligned}
\varsigma_{t+1}^{2} \mid \varsigma_{t}^{2} & \sim \operatorname{ARG}(\rho, c, \nu), \\
x_{t+1}-\phi_{\varsigma} \varsigma_{t+1}^{2} \mid z_{t} & \sim N\left(K_{0}+K_{1 \varsigma} \varsigma_{t}^{2}+K_{1 x} x_{t}, H_{0 x}+\sum_{i=1}^{M} H_{i x} \varsigma_{i t}^{2}\right) .
\end{aligned}
$$

See Appendix B for a more detailed construction of the density $f\left(\varsigma_{t+1}^{2} \mid \varsigma_{t}^{2}\right)$ and the definition of the parameters $(\rho, c, \nu)$.

The ARG distribution is the discrete-time counterpart to the multivariate square-root diffusion $\left(A_{M}(M)\right.$ process under $\mathbb{P}$ of Dai and Singleton (2000)). Several recent ETSMs 
(e.g., Bollerslev, Tauchen, and Zhou (2009) and Drechsler and Yaron (2011)) assume that the innovation in $\varsigma^{2}$ is Gaussian, a specification that is logically inconsistent with the nonnegativity of conditional variances. In contrast, by adopting an $A R G$ process: ${ }^{9}$ (i) $\varsigma_{t}^{2}$ is a non-negative process that solely governs the conditional volatility of the entire state vector $z_{t+1}$; and (ii) the conditional mean of $\varsigma_{t+1}^{2}$ is affine in $\varsigma_{t}^{2}\left(E_{t}\left[\varsigma_{t+1}^{2}\right]=\rho \varsigma_{t}^{2}+\nu c\right.$.) The positive semi-definite matrices $H_{0 x}$ and $H_{i x}(i=1, \ldots, M)$ govern the time-varying volatility of $x$.

For econometric identification we normalize $z$ so that, without loss of generality, $x_{t}$ and $\varsigma_{t}^{2}$ are conditionally independent $\left(\phi_{\varsigma}=0\right)$. Additionally, the intercepts $K_{0}$ in (17) are normalized to zero; and $B_{1}$, the loadings on $x_{t}$ for the yield on a one-period bond, are normalized to the row vector of ones. Further, we rotate $x$ so that $K_{1 x}$ has the Jordan form; and $c$ is fixed at $\frac{1}{2} \Delta t$. Finally, to prevent $\varsigma_{t}^{2}$ from being absorbed at zero we require that $\nu \geq 1$. The joint density of $\left(x_{t}, \varsigma_{t}^{2}\right)$ given by (16) and (17) gives the conditional density of $\left(\mathcal{P}_{x t}, \mathcal{P}_{\varsigma t}\right)$, after a Jacobian adjustment implied by (13) and (15). This is one of many equivalent normalization schemes that ensure that the extracted $\varsigma_{t}^{2}$ span the $e r_{t}^{h}(n)$.

Having characterized the joint density of the $N$ yield portfolios $\left(\mathcal{P}_{x t}, \mathcal{P}_{\varsigma t}\right)$, the construction of the likelihood function for all $J$ yields $y_{t}$ is completed by including an additional $J-N$ yield portfolios $\mathcal{P}_{e t}=W^{e} y_{t}$, with $W^{e}$ linearly independent of $W$, that are measured with additive errors. For simplicity we assume that these portfolios are observed with i.i.d. errors with zero means and common variance:

$$
\mathcal{P}_{e t}-W^{e} B_{\mathcal{P}} \mathcal{P}_{x t}-W^{e} \phi_{x}\left(A+C \varsigma_{t}^{2}\right) \sim N\left(0, \sigma_{e}^{2} I_{J-N}\right) .
$$

The parameter set of the model is $\left(A, C, \rho, \nu, K_{1 \varsigma}, K_{1 x}, H_{0 x}, H_{i x}, \sigma_{e}\right)$. Estimates of these parameters will serve as inputs into goodness-of-fit tests of affine ETSMs that are robust to specification of the remaining economic structure of these ETSMs.

\section{The Information in Long-Maturity Yields}

Prior to our empirical analysis we digress briefly and examine whether the range of maturities used affects estimates of expected excess returns in Treasury bond markets. Though it seems natural to include long maturity yields in an analysis of ETSMs, the high degree of co-movement among yields of different maturities may render some maturities redundant. Cochrane and Piazzesi (2005) and Duffee (2011), in their studies of risk premiums, chose to focus on maturities out to five years.

To shed light on whether longer maturity yields contain non-redundant information about risk premiums, we estimate the unconstrained linear projections of realized excess returns $x r_{t+h}^{h}(\tau)$ onto yield-curve information at date $t$. We set $h$ to six or twelve months and consider three choices of conditioning information: (i) yields on maturities from one to five years $(J=5)$; (ii) yields on maturities from one to five years plus the seven-, eight-, and ten-years $(J=8)$; and (iii) yields on maturities from one to ten years $(J=10)$. Of interest is the incremental predictive power of the latter cases $(J=8$ and $J=10)$ relative to when only yields up to five years are used in prediction.

\footnotetext{
${ }^{9}$ The following derivations are easily modified for other choices of non-negative affine distributions for $\varsigma_{t}^{2}$.
} 


\begin{tabular}{|c|c|c|c|c|c|c|c|c|}
\hline & \multicolumn{8}{|c|}{ Panel A: Six-Month Holding Period } \\
\hline & \multicolumn{2}{|c|}{$\mathrm{R}(1-5)$} & \multicolumn{3}{|c|}{$\mathrm{R}(1-5,7,8,10)$} & \multicolumn{3}{|c|}{$\mathrm{R}(1-10)$} \\
\hline & $\overline{\operatorname{Adj} R^{2}}$ & $\mathrm{BIC}$ & $\operatorname{Adj} R^{2}$ & pval & $\mathrm{BIC}$ & $\operatorname{Adj} R^{2}$ & pval & $\mathrm{BIC}$ \\
\hline mean $(x r)$ & 0.26 & -10.51 & 0.29 & 0.01 & -10.62 & 0.31 & 0.00 & $-10.63^{*}$ \\
\hline $\operatorname{xr}(3)$ & 0.28 & -13.56 & 0.32 & 0.00 & $-13.74^{*}$ & 0.32 & 0.00 & -13.66 \\
\hline $\operatorname{xr}(5)$ & 0.25 & -10.69 & 0.30 & 0.00 & $-10.88^{*}$ & 0.31 & 0.00 & -10.83 \\
\hline $\operatorname{xr}(7)$ & 0.25 & -9.04 & 0.27 & 0.04 & $-9.09^{*}$ & 0.29 & 0.00 & -9.09 \\
\hline \multirow[t]{4}{*}{$\operatorname{xr}(10)$} & 0.20 & -7.12 & 0.26 & 0.02 & -7.23 & 0.29 & 0.00 & $-7.31^{*}$ \\
\hline & \multicolumn{8}{|c|}{ Panel B: Twelve-Month Holding Period } \\
\hline & \multicolumn{2}{|c|}{$\mathrm{R}(1-5)$} & \multicolumn{3}{|c|}{$\mathrm{R}(1-5,7,8,10)$} & \multicolumn{3}{|c|}{$\mathrm{R}(1-10)$} \\
\hline & $\overline{\operatorname{Adj} R^{2}}$ & $\mathrm{BIC}$ & $\overline{\operatorname{Adj} R^{2}}$ & pval & $\mathrm{BIC}$ & $\overline{\operatorname{Adj} R^{2}}$ & pval & $\mathrm{BIC}$ \\
\hline $\operatorname{mean}(\mathrm{xr})$ & 0.38 & -8.55 & 0.42 & 0.02 & -8.79 & 0.44 & 0.00 & $-8.81^{*}$ \\
\hline $\operatorname{xr}(3)$ & 0.35 & -12.25 & 0.40 & 0.02 & $-12.60^{*}$ & 0.40 & 0.04 & -12.54 \\
\hline $\operatorname{xr}(5)$ & 0.36 & -9.21 & 0.42 & 0.00 & $-9.58^{*}$ & 0.43 & 0.00 & -9.55 \\
\hline $\operatorname{xr}(7)$ & 0.38 & -7.60 & 0.42 & 0.05 & -7.82 & 0.43 & 0.04 & $-7.82^{*}$ \\
\hline $\operatorname{xr}(10)$ & 0.36 & -5.69 & 0.41 & 0.02 & -5.89 & 0.43 & 0.00 & $-5.95^{*}$ \\
\hline
\end{tabular}

Table 2: Adjusted $R^{2}\left(\operatorname{Adj} R^{2}\right)$ from regressing six- (Panel A) and twelve-month (Panel B) excess returns $\mathrm{xr}(\mathrm{n})$ of bonds with $\mathrm{n}$ years to maturity on yields with 1-5 years to maturity ( $\mathrm{R}(1-5)), 1-10$ years to maturity $(\mathrm{R}(1-10))$, and $1-5,7,8,10$ years to maturity $(\mathrm{R}(1-5,7,8,10))$. Yields are extracted from the UFB dataset. pval's are for the joint significance tests of the loadings on longer-than-5-year maturities. The models chosen by the BIC scores (divided by 100) are indicated by an "*."

Using the CRSP treasury bond data and similar algorithms as described by Fama and Bliss (1987), we construct a consistent set of "Fama-Bliss" zero yields out to ten years to maturity through to the end of 2007 (the UFB dataset). Our sample period starts in January, 1984 after the abandonment of the monetary policy experiment between 1979 and 1982. To avoid the extreme market conditions of the ongoing crisis, we end our sample in December, 2007. BIC (Schwarz (1978)) scores are used to select the preferred forecasting model among these three specifications.

The adjusted $R^{2}$ 's from the projections are reported in Table 2, along with the probability values (pvals) of the chi-square tests of joint significance of the yields with maturities beyond five years. From these results it is clear that long maturity yields contain substantial extra predictive power over and above the first five yields. For example, for the annual holding period and the cross-sectional average of the excess returns (mean $(x r))$, the adjusted $R^{2}$ increases by $4 \%(6 \%)$ to $42 \%$ (44\%) by including three (five) longer-maturity yields. Moreover, for both this average and the individual excess returns, the BIC selection criterion always chooses information sets that include the long-maturity yields.

We explored the robustness of these findings in two ways. First, we re-estimated the projections including yields back to March, 1972. Earlier data was discarded owing to the relative sparseness of long-maturity bonds. Using this longer data set there is even stronger 


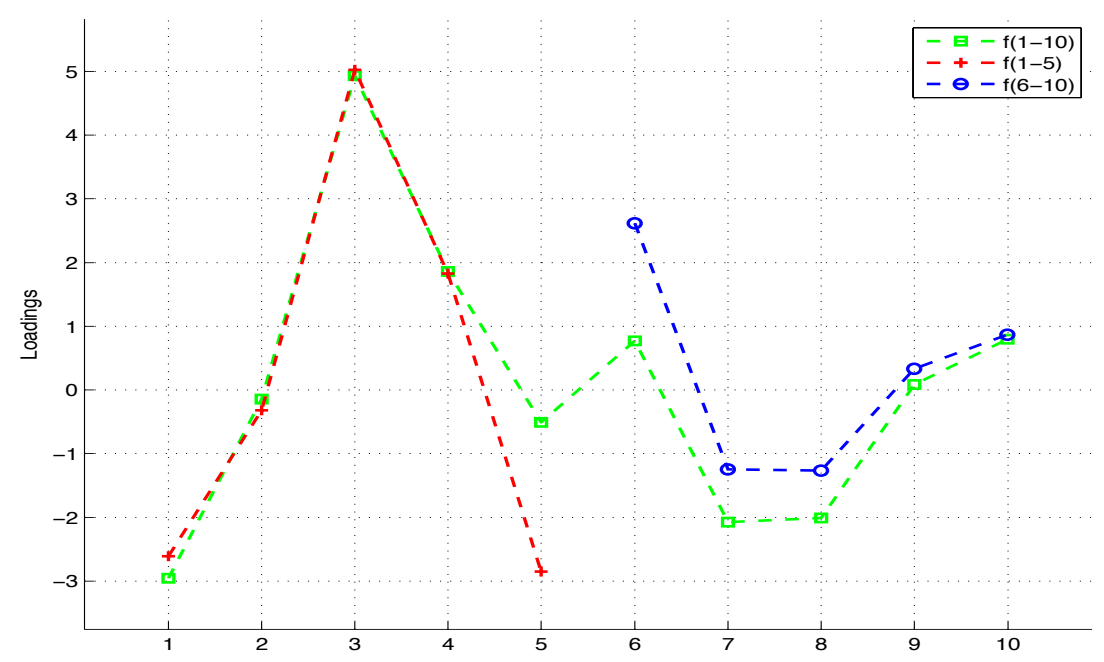

Figure 1: Loadings from the projections of mean $\left(x r_{t+h}\right)$ with $h=12$ (annual holding period) onto the first five one year forward rates $(\mathrm{f}(1-5))$, the first ten one-year forward rates $(\mathrm{f}(1-10))$, and the set of forward rates from 5 years to 6 years, 6 years to 7 years, ... 9 years to 10 years $(\mathrm{f}(6-10))$. The UFB data are used to construct forward rates and excess returns.

evidence that long-maturity yields have predictive content for excess returns in Treasury markets. Second, we downloaded the GSW dataset from the Federal Reserve's website and estimated comparable prediction equations. ${ }^{10}$ For the long sample, the longer yields embodied much less incremental forecasting power relative to the UFB data. For the shorter sample period the patterns were comparable, but the BIC criterion selected the specification $J=5$ for both the mean $(x r)$ and the excess returns on the seven- through ten-year bonds. That long-term yields have less incremental information about excess returns in the GSW data appears to be a consequence of the strong smoothing of forward rates in the underlying spline.

The weights on forward rates in the projections with UFB data are displayed in Figure 1. Clearly visible is the "tent-shape" pattern of loadings for the first five forward rates documented by Cochrane and Piazzesi (2005). The pattern of these loadings is essentially unchanged when all ten forward rates are included as predictors. Interestingly, the loadings on the long-maturity forwards for years six through ten form an inverted "tent-shape" pattern which is also robust to whether the first five forward rates are included or not.

\section{$5 \quad$ Volatility Factors and Expected Excess Returns}

In this section, we explore the robust implication RIETSM of ETSMs using historical bond yields and survey forecasts. When the volatility factors $\varsigma_{t}^{2}$ are fully spanned we can, without

\footnotetext{
${ }^{10}$ http://www.federalreserve.gov/Pubs/feds/2006/200628/200628abs.html
} 
loss of generality, base our empirical analysis on the spanning vector $V_{t} \equiv \mathcal{P}_{\varsigma t}-W_{\varsigma} B_{\mathcal{P}} \mathcal{P}_{x t}=$ $\beta \mathcal{P}_{t}$. Recall that $\mathcal{P}_{s t}$ corresponds to the first $M$ entries of $\mathcal{P}_{t}$ and, hence, the first $M \times M$ block of the $M \times N$ matrix $\beta$ is the identity matrix.

Most empirical studies of arbitrage-free term structure models have assumed that $N \leq 4$ and, as summarized in Table 1, this is also the case of many ETSMs. For our empirical analysis we set $N=4$ and $M=2$. This means that the set of volatility factors underlying the time variation in expected excess returns will also be $\mathcal{R}=2$, again consistent with much of the extant literature.

While the information sets generated by $z_{t}$ and any $N$ linearly independent yield portfolios $\mathcal{P}_{t}$ are (according to ETMSs) theoretically identical, there is the issue in practice of accurate measurement of yields (pricing of bonds). For instance, even though in theory $z_{t}$ is spanned by $y_{t}$, the sample projections of realized excess returns onto the information set generated by the observed yields $y_{t}^{o}$ are in general consistent estimators of their true theoretical counterparts only when $y_{t}^{o}$ is priced (nearly) perfectly by the ETSMs. It is now standard practice to accommodate measurement errors on all bond yields and to using filtering in estimation of macro-finance DTSMs. The errors $y_{t}^{o}-y_{t}$ can be large in macro-finance DTSMs, especially when $N$ is small (Joslin, Le, and Singleton (2012)).

Fortunately for our purposes the diversification that comes from using "portfolios" of yields, and in particular from setting $\mathcal{P}$ to the first $N P C$ s of bond yields, substantially mitigates these measurement issues. Joslin et al. (2012) show that the Kalman filter estimates of the model-implied low-order PCs in reduced-form DTSMs are (nearly) identical to their observed counterparts, even when the model-implied pricing errors $y_{t}^{o}-y_{t}$ become large. With this evidence in mind, we exploit the theoretical equivalence of the information sets generated by $z_{t}$ and $\mathcal{P}_{t}$ and conduct our analysis using the $P C \mathrm{~s} \mathcal{P}_{t}$.

Setting $N=4$, the joint distribution of $\mathcal{P}_{t}$ is determined by the joint density (16) - (17) along with the relevant Jacobian, as described in Section 3. The remaining $J-4 P C$ s of the $J$ yields $y_{t}, \mathcal{P}_{\text {et }}$, are assumed to priced up to additive errors according to (18). With these distributions in hand, and after imposing normalizations, we estimate the parameters by quasi-maximum likelihood $(Q M L)$. The resulting estimates of the free parameters in the $2 \times 4$ matrix $\beta$ are

$$
\beta=\left[\begin{array}{cccc}
1 & 0 & 10.48 & 58.66 \\
& & (0.243) & (0.078) \\
0 & 1 & -0.780 & -14.87 \\
& & (0.329) & (1.78)
\end{array}\right]
$$

where robust standard errors are given in parentheses. All four parameters are estimated with considerable precision. This was anticipated owing to the fact that the last $2 \times 2$ block of $\beta$ is fully determined by the Jordan form of $K_{1 x}$ which, in turn, is identified primarily from the cross-sectional restrictions in (10). ${ }^{11}$

Equipped with a precise estimate of $\beta$, we proceed to examine the following implications of ETSMs: (i) $V_{t}$ fully captures the information about volatility spanned by yields; (ii) after

\footnotetext{
${ }^{11}$ By way of contrast, the loadings from the time-series projection of squared residuals (from the projection of $P C 1_{t+1}$ onto $\mathcal{P}_{t}$ ) onto $\mathcal{P}_{t}$ are estimated much less precisely.
} 


\begin{tabular}{|c|c|c|c|c|c|c|c|c|c|}
\hline & \multicolumn{3}{|c|}{$H=2$} & \multicolumn{3}{|c|}{$H=4$} & \multicolumn{3}{|c|}{$H=6$} \\
\hline & \multicolumn{2}{|c|}{ Adj. $R^{2}$} & \multirow[b]{2}{*}{ pval } & \multicolumn{2}{|c|}{ Adj. $R^{2}$} & \multirow[b]{2}{*}{ pval } & \multicolumn{2}{|c|}{ Adj. $R^{2}$} & \multirow[b]{2}{*}{ pval } \\
\hline & $4 \mathrm{PCs}$ & $V_{t}$ & & $4 \mathrm{PCs}$ & $V_{t}$ & & $4 \mathrm{PCs}$ & $V_{t}$ & \\
\hline PC1 & $0.080^{* * *}$ & $0.083^{* * *}$ & 0.877 & $0.072^{* * *}$ & $0.071^{* * *}$ & 0.466 & $0.056^{* * *}$ & $0.059^{* * *}$ & 0.771 \\
\hline $\mathrm{PC} 2$ & $0.031^{* *}$ & 0.021 & 0.571 & $0.037^{* *}$ & 0.035 & 0.797 & $0.040^{* * *}$ & $0.042^{* *}$ & 0.914 \\
\hline PC3 & $0.036^{* * *}$ & 0.022 & 0.241 & $0.039^{* *}$ & $0.041^{*}$ & 0.848 & $0.042^{* *}$ & $0.041^{*}$ & 0.637 \\
\hline PC4 & $0.150^{* * *}$ & $0.147^{* * *}$ & 0.620 & $0.157^{* * *}$ & $0.157^{* * *}$ & 0.737 & $0.126^{* * *}$ & $0.127^{* * *}$ & 0.629 \\
\hline
\end{tabular}

Table 3: Comparison of projections of squared forecast errors, obtained from a $V A R(1)$ model of $\mathcal{P}_{t+1}$, onto $\mathcal{P}_{t}$ versus $V_{t}$. "pval" is the robust probability value for the chi-square test of the null hypothesis $\beta_{\mathcal{P}}=\beta_{V} \beta$. The superscripts $\left({ }^{*},{ }^{* *},{ }^{* * *}\right)$ denote the level of significance (at $10 \%, 5 \%, 1 \%$, respectively) of the $\chi^{2}$ test of joint significance of the regression coefficients (excluding intercepts).

accounting for $V_{t}$, information in the yield curve has no predictive content for realized excess returns; and (iii) after accounting for $V_{t}$, information in the yield curve has no predictive content for the conditional volatilities of inflation and consumption growth.

\section{Does $V_{t}$ fully capture spanned yield volatility?}

When volatility is spanned, the transformed volatility factors $V_{t}$ span the conditional variances of the state $z_{t}$. To assess whether this is in fact the case we estimate the projections

$$
E_{t}\left(\epsilon_{t+H}^{2}\right)=\mathrm{constant}+\beta_{\mathcal{P}} \mathcal{P}_{t} \text { and } E_{t}\left(\epsilon_{t+H}^{2}\right)=\mathrm{constant}+\beta_{V} V_{t}
$$

for $H=2,4,6$ (in months), where $\epsilon_{t}$ denotes the error in forecasting $\mathcal{P}_{t}$ based on information at time $t-1$. An implication of RIETSM and the autonomous nature of $V_{t}$ under $\mathbb{P}$ is that the second moments $E_{t}\left[\epsilon_{t+j}^{2}\right]$, for all $j>1$, depend solely on $V_{t}$. $\mathcal{P}_{t}$ follows an affine process under $\mathbb{P}$, so consistent estimates of the forecasting errors $\epsilon_{t}$ are obtained by fitting a $V A R(1)$ model for $\mathcal{P}_{t}$. In Table 3 we report the adjusted $R^{2}$ statistics of the two sets of projections in (20) for each of the first four $P C$ s comprising $\mathcal{P}_{t}$.

Across all horizons $H$ and all $P C \mathrm{~s}^{-}$- particularly $P C$, arguably the most important driver of time varying volatility in yields $-V_{t}$ captures most of the volatility information spanned by the $P C$ s. For example, for $H=6$ and $P C 1$, the adjusted $R^{2}$ from conditioning on $\mathcal{P}_{t}\left(V_{t}\right)$ is $5.6 \%(5.9 \%)$. To formally evaluate the differences in fits, we conduct $\chi^{2}$ tests of the null hypothesis $\beta_{\mathcal{P}}=\beta_{V} \beta$ implied by ETSMs. ${ }^{12}$ The probability values ("pvals") confirm that the small differences in $R^{2}$ s are statistically insignificant.

We next consider an alternative approach to constructing the forecast error $\epsilon_{t}$ that uses the Blue-Chip financial survey forecasts $(\mathrm{BCFF})$ of interest rates in place of the conditional means

\footnotetext{
${ }^{12}$ The probability values of this test reported in Table 3 are robust to the sequential nature of our estimation. Specifically, we account for the use of first-stage estimates of $\beta$ as well as $\epsilon_{t}$ in estimating the regressions in (20). To account for the serial correlation of errors, we use the Newey-West estimates of the large-sample variance matrix with twelve lags.
} 
estimated by linear projection. ${ }^{13}$ We presume that the construction of $\epsilon_{t}$ from the BCFF data is robust to the presence of information that has forecasting power for future yields and that is unspanned by $\mathcal{P}_{t} .{ }^{14}$ Such unspanned risks would contaminate the $V A R(1)$-implied conditional means (based on yields alone) and the associated volatility regressions in (20).

Since the BCFF forecasts are for three-month average yields, ${ }^{15}$ at each time $\mathrm{t}$ we let

$$
Q_{h, t}^{(i)}=E_{t}^{(i)}\left[y_{t+h}+y_{t+h+1}+y_{t+h+2}\right]
$$

denote the h-month ahead average yield forecast formed by forecaster $i{ }^{16}$ For each horizon $h$, the difference $Q_{h-1, t+1}^{(i)}-Q_{h, t}^{(i)}$ is the revision or surprise by forecaster $i$. After trimming out extreme forecasts, we construct our aggregate measure of surprise by averaging the squared surprises across all forecasters and summing over all forecast horizons

$$
\epsilon_{t+1}^{2}=\sum_{h} \frac{1}{N_{h, t}} \sum_{i}\left(Q_{h-1, t+1}^{(i)}-Q_{h, t}^{(i)}\right)^{2},
$$

where $N_{h, t}$ is the number of forecasters for horizon $h$ and we include the horizons $h=9,12,15$, and 18 months out. Affine ETSMs imply that the conditional second moments of the surprises $\epsilon_{t+1}$ are affine in $V_{t}$. Accordingly, for each yield maturity, we compare the projections

$$
E_{t}\left(\epsilon_{t+H}^{2}\right)=\text { constant }+\beta_{\mathcal{P}} \mathcal{P}_{t} \text { and } E_{t}\left(\epsilon_{t+H}^{2}\right)=\text { constant }+\beta_{V} V_{t},
$$

again for $H=2,4$, and 6 . We omit $H=1$, because the BCFF surveys are conducted over a two-day period somewhere between the 20th and 26th of a given month $\mathrm{t}$ and so $e_{t+1}$ is not, strictly speaking, a surprise relative to the information set at the end of month $t$.

The adjusted $R^{2}$ s for the projections in (21) are reported in Table 4 for maturities $n=$ 3 months, 1, 3, 5, 7, and 10 years. Across these maturities and all choices of $H$, the second moments conditioned on $V_{t}$ and $\mathcal{P}_{t}$ are strikingly similar and, in fact, in many cases the projections based on $V_{t}$ have larger adjusted $R^{2}$ s. It is therefore not surprising that the null hypothesis that $V_{t}$ captures all of the forecasting power of $\mathcal{P}_{t}$ typically cannot be rejected. Note that the estimates of $\beta_{\mathcal{P}}$ and $\beta_{V}$ are jointly significant for almost all choices of maturity and $H$. Therefore the similar forecasting power between $V_{t}$ and $\mathcal{P}_{t}$ is not simply due to a lack of predictive power from these conditioning variables.

\section{Does $V_{t}$ fully span expected excess returns conditioned on $\mathcal{P}_{t}$ ?}

Having documented that $V_{t}$ essentially subsumes the volatility information embodied in $\mathcal{P}_{t}$, we next examine whether $V_{t}$ also spans expected excess returns conditioned on $\mathcal{P}_{t}$. Comparisons

\footnotetext{
${ }^{13}$ The Blue Chip forecasts were obtained from Wolters Kluwer.

${ }^{14}$ The descriptive analysis of Ludvigson and Ng (2010) identifies such unspanned macro risks and Joslin, Priebsch, and Singleton (2011) develop a DTSM that accommodates such unspanned risks.

${ }^{15}$ Except for the three-month and six-month maturities, the BCFF forecasts are for averages of par yields. See Appendix A for details of the construction of zero yield forecasts. Additionally, the BCFF forecasts are over calendar quarters. We follow the interpolation approach of Chun (2010) to build forecasts for non-calendar quarters. The same interpolation technique is used to construct forecasts for horizons not provided by the BCFF newsletter.

${ }^{16}$ The one- and two-quarter forecasts are highly volatile and therefore omitted in our calculations.
} 


\begin{tabular}{|c|c|c|c|c|c|c|c|c|c|}
\hline & \multicolumn{3}{|c|}{$H=2$} & \multicolumn{3}{|c|}{$H=4$} & \multicolumn{3}{|c|}{$H=6$} \\
\hline & \multicolumn{2}{|c|}{$\operatorname{Adj} R^{2}$} & \multirow[b]{2}{*}{ pval } & \multicolumn{2}{|c|}{$\operatorname{Adj} R^{2}$} & \multirow[b]{2}{*}{ pval } & \multicolumn{2}{|c|}{$\operatorname{Adj} R^{2}$} & \multirow[b]{2}{*}{ pval } \\
\hline & $4 \mathrm{PCs}$ & $V_{t}$ & & $4 \mathrm{PCs}$ & $V_{t}$ & & $4 \mathrm{PCs}$ & $V_{t}$ & \\
\hline $3 \mathrm{~m}$ & $0.227^{* * *}$ & $0.206^{* * *}$ & 0.617 & $0.250^{* * *}$ & $0.219^{* * *}$ & 0.439 & $0.266^{* * *}$ & $0.230^{* * *}$ & 0.281 \\
\hline $1-y$ & $0.221^{* *}$ & $0.215^{* * *}$ & 0.918 & $0.264^{* * *}$ & $0.244^{* * *}$ & 0.721 & $0.267^{* * *}$ & $0.242^{* * *}$ & 0.742 \\
\hline $3-y$ & $0.248^{* * *}$ & $0.243^{* * *}$ & 0.798 & $0.294^{* * *}$ & $0.266^{* * *}$ & 0.313 & $0.295^{* * *}$ & $0.270^{* * *}$ & 0.214 \\
\hline $5-y$ & $0.226^{* * *}$ & $0.227^{* * *}$ & 0.950 & $0.260^{* * *}$ & $0.249^{* * *}$ & 0.617 & $0.261^{* * *}$ & $0.249^{* * *}$ & 0.508 \\
\hline 7-y & $0.271^{* * *}$ & $0.266^{* * *}$ & 0.750 & $0.305^{* * *}$ & $0.287^{* * *}$ & 0.333 & $0.308^{* * *}$ & $0.287^{* * *}$ & 0.191 \\
\hline $10-y$ & $0.275^{* * *}$ & $0.269^{* * *}$ & 0.724 & $0.311^{* * *}$ & $0.296^{* * *}$ & 0.340 & $0.317^{* * *}$ & $0.295^{* * *}$ & 0.205 \\
\hline
\end{tabular}

Table 4: Regressions of squared residuals constructed from BCFF yield forecasts on PCs and $V_{t}$. The superscripts $\left({ }^{*},{ }^{* *},{ }^{* *}\right)$ denote the level of significance (at $10 \%, 5 \%, 1 \%$, respectively) of the $\chi^{2}$ test of joint significance of the regression coefficients (not including intercepts).

of the projections of realized excess returns onto $V_{t}$ and $\mathcal{P}_{t}$ are displayed in Table 5 for holding periods of lengths $h=3,6$, and 12 months.

Overall, $V_{t}$ seems to capture a substantial amount of predictability spanned by $\mathcal{P}_{t}$, particularly for longer maturity bonds. For example, the adjusted $R^{2}$ from regressing 12 month excess returns on a 10 -year bond on $\mathcal{P}_{t}$ is $32.5 \%$, compared to $28.1 \%$ when $V_{t}$ is used as the regressor. This difference is not statistically significant (pval $=0.148$ ). Nevertheless, there is strong evidence that, for shorter maturity bonds, there is predictive information in yields that is not fully captured by $V_{t}$. The 3-month and 6-month excess returns on a 1-year zero are predicted by $\mathcal{P}_{t}$ with adjusted $R^{2}$ s of $16.5 \%$ and $31.3 \%$, compared to $9.9 \%$ and $21.3 \%$ by $V_{t}$. For both cases, the pvals of the difference tests are smaller than $1 \%$.

That $V_{t}$ does not fully account for the predictive content of $\mathcal{P}_{t}$ is anticipated by studies that show significant effects of bond supplies, foreign demands, and clienteles on the shape of the U.S. Treasury curve (e.g., Greenwood and Vayanos (2010b)). Another influence on the shape of the intermediate segment of the Treasury yield curve was the hedging activities of mortgage traders (Duarte (2008)). Notwithstanding these considerations, it is notable how much of the variation in risk premiums is captured by the two-dimensional $V_{t}$ extracted from the volatility structure of treasury yields.

\section{Does $V_{t}$ represent spanned volatility of inflation or real growth?}

Most ETSMs that price nominal bonds have assumed that the conditional variance of inflation is spanned by the same volatility factors that determine the variances of bond yields, $V_{t}$ in our setting. For example, a simplified structure of shocks to yields $\left(y_{t}\right)$ and inflation $\left(\pi_{t}\right)$ in Bansal and Shaliastovich (2010) can be written as:

$$
\begin{aligned}
\pi_{t+1}-E_{t}\left[\pi_{t+1}\right] & =\phi_{\pi g} \sigma_{g t} \eta_{t+1}+\phi_{\pi x} \sigma_{x t} e_{t+1}, \\
y_{t+1}-E_{t}\left[y_{t+1}\right] & =\phi_{y g} \sigma_{g t} \eta_{t+1}+\phi_{y x} \sigma_{x t} e_{t+1},
\end{aligned}
$$




\begin{tabular}{|c|c|c|c|c|c|c|c|c|c|}
\hline & \multicolumn{3}{|c|}{$h=3$ months } & \multicolumn{3}{|c|}{$h=6$ months } & \multicolumn{3}{|c|}{$h=12$ months } \\
\hline & \multicolumn{2}{|c|}{ Adj. $R^{2}$} & \multirow[b]{2}{*}{ pval } & \multicolumn{2}{|c|}{ Adj. $R^{2}$} & \multirow[b]{2}{*}{ pval } & \multicolumn{2}{|c|}{ Adj. $R^{2}$} & \multirow[b]{2}{*}{ pval } \\
\hline & $4 \mathrm{PCs}$ & $V_{t}$ & & $4 \mathrm{PCs}$ & $V_{t}$ & & $4 \mathrm{PCs}$ & $V_{t}$ & \\
\hline mean $(x r)$ & 0.088 & 0.063 & 0.159 & 0.250 & 0.196 & 0.081 & 0.367 & 0.315 & 0.379 \\
\hline $\operatorname{xr}(1)$ & 0.165 & 0.099 & 0.004 & 0.313 & 0.213 & 0.003 & NA & NA & NA \\
\hline $\operatorname{xr}(3)$ & 0.113 & 0.077 & 0.134 & 0.267 & 0.201 & 0.089 & 0.371 & 0.287 & 0.122 \\
\hline $\operatorname{xr}(5)$ & 0.086 & 0.055 & 0.131 & 0.262 & 0.196 & 0.060 & 0.375 & 0.312 & 0.275 \\
\hline $\operatorname{xr}(7)$ & 0.096 & 0.071 & 0.167 & 0.253 & 0.199 & 0.073 & 0.369 & 0.316 & 0.271 \\
\hline $\operatorname{xr}(10)$ & 0.054 & 0.042 & 0.424 & 0.190 & 0.153 & 0.124 & 0.325 & 0.281 & 0.148 \\
\hline
\end{tabular}

Table 5: Projections of bonds excess returns onto $\mathcal{P}_{t}$ and $V_{t}$ for holding periods of length 3,6 , and 12 months on bonds of maturities 1,3,5,7, and 10 years. mean $(x r)$ is the cross-sectional average of the excess returns.

where $\eta_{t+1}$ and $e_{t+1}$ are necessarily uncorrelated (to preserve the affine structure of bond yields). It is clear that the conditional variances of inflation and yields are both linear functions of $\sigma_{g t}^{2}$ and $\sigma_{x t}^{2}$ - elements of $V_{t}$ in our setting.

Moreover this model, and indeed most affine $E T S M \mathrm{~s}$, are first and foremost real business cycle models in the sense that it is the volatilities of real side of the economy ( $L R R$, consumption growth, surplus consumption) that are assumed to drive time-varying volatility in inflation (if present at all). In such models, $V_{t}$ derived from yields must be as powerful as $\mathcal{P}_{t}$ in predicting squared inflation forecast errors. Moreover, structures similar to (22) and (23) also reveal that the conditional covariances between inflation and yields must be linear in $V_{t}$. Therefore another implication of these models is that $\mathcal{P}_{t}$ and $V_{t}$ must be equally powerful in predicting the product of yield and inflation forecast errors.

There is extensive evidence of time-varying volatility of inflation (see, for examples, Engle (1982) and Stock and Watson (2007)). Piazzesi and Schneider (2007), Rudebusch and Wu (2007), Doh (2011), and Wright (2011) argue, within the context of affine term structure models, that a decline in inflation uncertainty was partially responsible for the decline in term premiums during the past twenty years. Doh, in particular, focuses on an ETSM with $L R R$ structured similarly to the model in Bansal and Shaliastovich (2010). A distinguishing feature of our analysis is that we link measures of inflation volatility directly to the factors $V_{t}$ extracted from the yield curve that the risk structure of ETSMs identify as the volatility factors that drive excess returns.

To explore these implications we construct inflation forecast errors with the Blue Chip Economic Indicator (BCEI) survey forecasts, using the approach described above. We again project the squared forecast errors onto $\mathcal{P}_{t}$ and $V_{t}$ as in (21) and compare the fits of the two sets of regressions. From the first row of Table 6 it is seen that $V_{t}$ captures almost all of the time variation in spanned inflation volatility. For example, for $H=2, \mathcal{P}_{t}$ and $V_{t}$ forecast squared average inflation residuals with adjusted $R^{2}$ s of $28.3 \%$ and $25.6 \%$, respectively, and the difference is not statistically significant.

Panel (a) of Figure 2 displays the fitted volatilities of inflation conditional on the yield- 


\begin{tabular}{|c|c|c|c|c|c|c|c|c|c|}
\hline & \multicolumn{3}{|c|}{$H=2$} & \multicolumn{3}{|c|}{$H=4$} & \multicolumn{3}{|c|}{$H=6$} \\
\hline & \multicolumn{2}{|c|}{ Adj. $R^{2}$} & \multirow[b]{2}{*}{ pval } & \multicolumn{2}{|c|}{ Adj. $R^{2}$} & \multirow[b]{2}{*}{ pval } & \multicolumn{2}{|c|}{ Adj. $R^{2}$} & \multirow[b]{2}{*}{ pval } \\
\hline & $4 \mathrm{PCs}$ & $V_{t}$ & & $4 \mathrm{PCs}$ & $V_{t}$ & & $4 \mathrm{PCs}$ & $V_{t}$ & \\
\hline$e_{C P I^{2}}$ & $0.283^{* * *}$ & $0.256^{* * *}$ & 0.130 & $0.334^{* * *}$ & $0.302^{* * *}$ & 0.066 & $0.376^{* * *}$ & $0.313^{\text {*** }}$ & 0.068 \\
\hline$e_{G D P^{2}}$ & $0.329^{* * *}$ & $0.276^{* * *}$ & 0.032 & $0.403^{* * *}$ & $0.336^{* * *}$ & 0.011 & $0.449^{* * *}$ & $0.355^{* * *}$ & 0.002 \\
\hline$e_{C P I} e_{G D P}$ & 0.031 & 0.024 & 0.828 & -0.005 & 0.000 & 0.190 & $0.006^{*}$ & 0.009 & 0.834 \\
\hline
\end{tabular}

Table 6: Regressions of squared inflation residuals $\left(e_{C P I}^{2}\right)$, squared real GDP residuals $\left(e_{G D P}{ }^{2}\right)$, and the product of inflation and real GDP residuals $\left(e_{C P I} e_{G D P}\right)$ on $\mathcal{P}_{t}$ and $V_{t}$.

curve extracted volatility factors $V_{t}$. Consistent with the view that inflation risk declined in the late 1980's and 1990's, there is a persistent decline in fitted volatility over this portion of our sample. Interestingly, while the downward trend is also visible in the univariate EGARCH-fitted volatility, the latter declines much more rapidly in the late 1980's and then the two measures catch up with each other a decade later. During the later portion of our sample the EGARCH estimates seem to stabilize whereas the $V$-implied volatilities are relatively more choppy (although they never hit the truncation point at zero.)

As noted above, the vast majority of ETSMs have the stronger property that timevarying volatility in inflation arrises from the real side of the economy from the volatilities of consumption growth and (when relevant) long-run risks. In such settings, $V_{t}$ should be the common source of time-varying inflation and real economic risks. No consensus has emerged on the degree of stochastic volatility in consumption growth. Early versions of ETSMs with LRR (e.g., Bansal and Yaron (2004)) assumed that time variation in risk premiums was induced entirely by the consumption volatility. Later, more flexible versions accommodated distinct stochastic volatility in the $L R R$ factor (conditional mean of consumption growth). Variation in the $L R R$ factor could in principle be an important source of variation in risk premiums even if consumption growth shows weak time-varying volatility, though detecting this from historical consumption data is inherently difficult (Hansen, Heaton, and Li (2008)). Under RIETSM, the yield curve should be informative about the total contribution of real-side risk to bond market risk premiums.

In the habit-based model of Campbell and Cochrane (1999) consumption growth is homoskedastic and the source of time-varying risk is stochastic volatility in the surplus consumption ratio. Wachter (2006) and Le, Singleton, and Dai (2010) adopted similar specifications of the volatility structure in their ETSMs. Again, the current yield curve should be informative about this real economic risk.

There is wide-spread concern about the reliability of aggregate consumption data in tests of dynamic asset pricing models, and in addition we do not have a monthly series of real consumption forecasts for our sample period. We can shed some light on connection between $V_{t}$ and real economic activity by examining the BCFF survey forecasts of real GDP growth and associated real GDP forecast errors (constructed using the same approach used for constructing inflation forecast errors). We again project the squared GDP forecast errors 


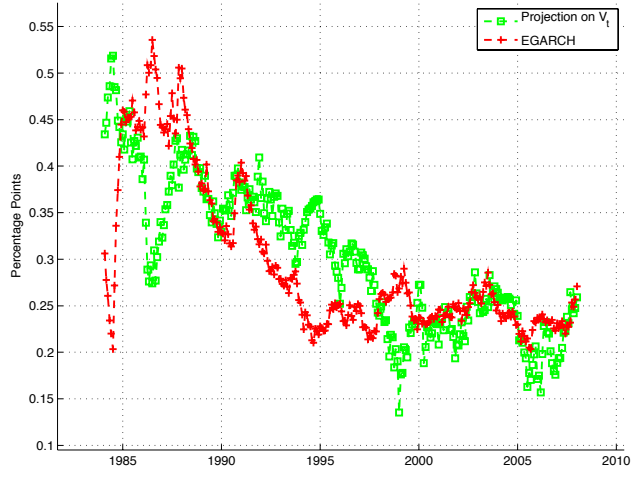

(a) Inflation Volatility

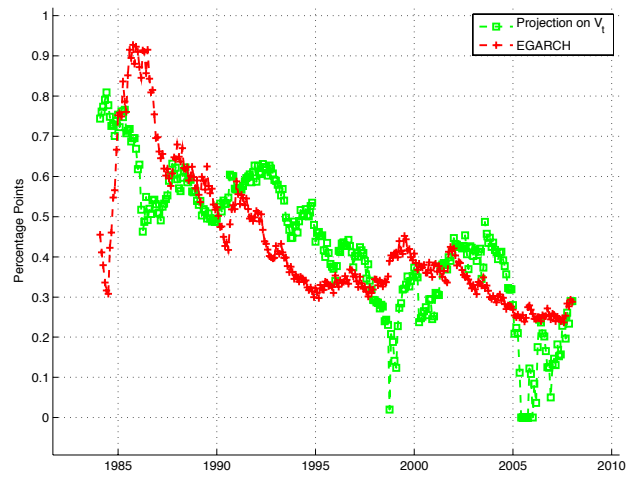

(b) GDP Volatility

Figure 2: Fitted conditional volatilities of inflation (Panel (a)) and GDP growth (Panel (b)) from projections of squared forecast errors onto the yield-curve extracted volatilities $V_{t}$. For comparison, each panel includes the fitted values from an $\operatorname{EGARCH}(1,1)$ model of volatility. Negative fitted values were truncated at zero.

onto $\mathcal{P}_{t}$ and $V_{t}$ as in (21) and compare the fits of the two sets of regressions.

Most notable about the second row of Table 6 is the finding that both $\mathcal{P}_{t}$ and $V_{t}$ have substantial predictive content for the volatility of real GDP growth, with adjusted $R^{2}$ ranging from $28 \%$ to $45 \%$. The $P C \mathrm{~s} \mathcal{P}_{t}$ always have more predictive power, yet $V_{t}$ accounts for roughly $80 \%$ of the conditional variation in real GDP growth that is spanned by yield $P C$ s. Panel B of Figure 2 plots the $V$-implied volatility estimates together with their EGARCH counterparts. The downward trend in both series is consistent with the "Great Moderation" observed by Stock and Watson (2002) and others.

Now real GDP growth is far from a perfect substitute for consumption growth- the object of interest in most ETSMs- since, among other reasons, consumption is allocated across both domestic and imported goods and services, whereas GDP measures domestic output. Nevertheless, the fitted conditional volatility of real GDP growth is a natural construct to focus on because GDP growth is a key ingredient in the Federal Reserves setting of monetary policy (e.g., through the "Taylor rule"). These linkages have been explored by, among others, McCallum (1994) and Gallmeyer, Hollifield, and Zin (2005) within affine ETSMs that explicitly incorporate the setting of a short-term rate by a monetary authority.

When we project the 3-, 6-, and 12-month average excess returns onto the fitted volatility of GDP from the second row of Table 6 , we get $R^{2}$ 's of $6 \%, 16 \%$, and $25 \%$, respectively. Using the fitted volatility of inflation as a predictor gives comparable (slightly lower) adjusted $R^{2}$ s. Thus, fitted volatility from the real side of the economy roughly matches the explanatory power of inflation risk for excess returns in bond markets, consistent with the presumption of many ETSMs. 


\begin{tabular}{|c|c|c|c|c|c|c|c|c|c|}
\hline & \multicolumn{3}{|c|}{$H=2$} & \multicolumn{3}{|c|}{$H=4$} & \multicolumn{3}{|c|}{$H=6$} \\
\hline & \multicolumn{2}{|c|}{$\operatorname{AdjR} 2$} & \multirow[b]{2}{*}{ pval } & \multicolumn{2}{|c|}{$\operatorname{AdjR} 2$} & \multirow[b]{2}{*}{ pval } & \multicolumn{2}{|c|}{ AdjR2 } & \multirow[b]{2}{*}{ pval } \\
\hline & $4 \mathrm{PCs}$ & $V_{t}$ & & $4 \mathrm{PCs}$ & $V_{t}$ & & 4 PCs & $V_{t}$ & \\
\hline \multicolumn{10}{|c|}{ Panel A: Inflation } \\
\hline $3 \mathrm{~m}$ & 0.180 & $0.171^{* * *}$ & 0.716 & $0.215^{* * *}$ & $0.198^{* * *}$ & 0.702 & $0.243^{* * *}$ & $0.220^{* * *}$ & 0.647 \\
\hline $1-y$ & $0.102^{* * *}$ & $0.104^{* * *}$ & 0.720 & $0.125^{*}$ & $0.113^{* * *}$ & 0.891 & $0.149^{* * *}$ & $0.135^{* * *}$ & 0.595 \\
\hline $3-y$ & $0.098^{* *}$ & $0.101^{* * *}$ & 0.625 & $0.124^{* * *}$ & $0.120^{* * *}$ & 0.848 & $0.152^{* * *}$ & $0.147^{* * *}$ & 0.688 \\
\hline $5-\mathrm{y}$ & $0.106^{* * *}$ & $0.109^{* * *}$ & 0.625 & $0.129^{* * *}$ & $0.124^{* * *}$ & 0.703 & $0.159^{* * *}$ & $0.153^{* * *}$ & 0.485 \\
\hline $7-\mathrm{y}$ & $0.108^{* *}$ & $0.110^{* * *}$ & 0.332 & $0.128^{* * *}$ & $0.124^{* * *}$ & 0.549 & $0.152^{* * *}$ & $0.145^{* * *}$ & 0.515 \\
\hline $10-\mathrm{y}$ & $0.117^{*}$ & $0.118^{* *}$ & 0.535 & $0.135^{* *}$ & $0.128^{* * *}$ & 0.641 & $0.156^{* * *}$ & $0.148^{* * *}$ & 0.600 \\
\hline \multicolumn{10}{|c|}{ Panel B: Real GDP } \\
\hline $3 \mathrm{~m}$ & $0.056^{* *}$ & $0.060^{* *}$ & 0.752 & $0.080^{*}$ & 0.077 & 0.848 & $0.098^{*}$ & 0.091 & 0.715 \\
\hline $1-\mathrm{y}$ & $0.022^{*}$ & $0.026^{*}$ & 0.939 & $0.039^{*}$ & 0.045 & 0.942 & $0.045^{* *}$ & 0.050 & 0.352 \\
\hline $3-\mathrm{y}$ & $0.007^{*}$ & 0.011 & 0.392 & 0.019 & 0.025 & 0.975 & $0.024^{* *}$ & 0.030 & 0.650 \\
\hline $5-\mathrm{y}$ & $0.024^{* *}$ & 0.028 & 0.130 & $0.038^{*}$ & 0.045 & 0.912 & $0.043^{*}$ & 0.048 & 0.874 \\
\hline $7-y$ & $0.035^{*}$ & $0.038^{*}$ & 0.073 & $0.052^{* * *}$ & $0.058^{* *}$ & 0.922 & $0.056^{* *}$ & $0.062^{*}$ & 0.986 \\
\hline $10-y$ & $0.036^{* *}$ & 0.040 & 0.682 & $0.052^{* * *}$ & $0.057^{* *}$ & 0.997 & $0.057^{* *}$ & $0.062^{*}$ & 0.999 \\
\hline
\end{tabular}

Table 7: Regressions of the products of CPI inflation forecast residuals and yield forecast residuals (Panel A) and the products of real GDP forecast residuals and yield forecast residuals (Panel B) on $\mathcal{P}_{t}$ and $V_{t}$.

Additional insights into the effects of real and inflation shocks on risk premiums come from inspection of the links between $V_{t}$ and the conditional covariances between yields, real GDP, and inflation. In the prototypical $L R R$ model, $V_{t}$ fully determines the conditional covariance between output growth and inflation. From the last row of Table 6 it is seen that neither $\mathcal{P}_{t}$ nor $V_{t}$ has predictive power for the product of the inflation and GDP residuals. In the light of our earlier findings that $V_{t}$ and $\mathcal{P}_{t}$ have strong predictive power for the conditional volatilities of these variables, the covariance results suggest that there is a canceling effect that renders the covariance largely time-invariant, at least for our conditioning set.

This finding, in turn, raises the possibility that conditional covariances between yields and GDP and yields and inflation may be very different. This is confirmed in Table 7 where we report the projections of the products of inflation and yield forecast errors (Panel A) and the products of real GDP forecast residuals and yield forecast residuals (Panel B) on $\mathcal{P}_{t}$ and $V_{t}$. Across all yield maturities, the conditional covariances between inflation and yields are quite strongly time-varying and well captured by $V_{t}$, as implied by ESTM's. Except for one instance, the $\chi^{2}$ statistics of the joint significance test of the regression coefficients are all significant at the $1 \%$ level.

In contrast, for the products between real GDP and yield forecast errors, the adjusted $R^{2}$ 's in Panel B are substantially lower than their counterparts in Panel A, and the corresponding $\chi^{2}$ test statistics are mostly insignificant. Evidently $V_{t}$ is substantially more informative about the conditional covariances between yields and inflation than about the covariances 
between yields and real GDP.

To help with the interpretation of these results note that, for conditionally Gaussian shocks and ignoring (largely negligible) Jensen terms,

$$
E_{t}\left[x r_{t+1}\right] \approx n \operatorname{Cov}_{t}\left(m_{t+1}^{R}, y_{n, t+1}\right)-n \operatorname{Cov}_{t}\left(\pi_{t+1}, y_{n, t+1}\right)
$$

where $m_{t+1}^{R}$ denotes agents' marginal rate of substitution for consumption (all but the term $-\pi_{t+1}$ in (3)). An implication of ETSMs is that that $V_{t}$ drives the time variation of both of the right-hand side terms. Thus, up to this approximation, the results in Table 7 indicate that inflation risks represent an important source of variation in bond risk premiums. On the other hand, our findings suggest that the first term is a relatively small contributor to risk premiums, though a more decisive verification of this requires better quality data on aggregate consumption than is currently available.

\section{6 "Unspanned" Risks and Excess Returns on Bonds}

A maintained assumption in virtually the entire literature on affine ETSMs is that the state of the economy is spanned by returns on bonds and certain equity claims. As noted in Section 1, this observation is central to many prior studies of $L R R$ and equity returns. However, for the bond market, there is an extensive literature documenting the existence of unspanned stochastic volatility (USV) (e.g., Collin-Dufresne and Goldstein (2002), Li and Zhao (2006), and Joslin (2011)). Since, within ETSMs satisfying RIETSM, the $\varsigma_{t}^{2}$ driving excess returns are the sources of time-varying volatility in yields, the presence of USV raises the important possibility that ETSMs have mis-specified the conditional distribution of $y_{t}$ in a way that may have distorted estimated risk premiums for bond markets.

\section{Further Evidence on the Predictability of Excess Returns}

Up to this point we have focused on the predictability of excess returns based on yields $\left(y_{t}\right)$, because ETSMs imply that $\varsigma_{t}^{2}$ (and hence the $e r_{t}^{h}(n)$ ) are spanned by $y_{t}$. Prior to exploring the implications of USV for ETSMs we examine whether other conditioning information has predictive content for returns. We can always decompose excess returns $\operatorname{er}_{t}^{h}(n)$ as

$$
\underbrace{n y_{n, t}-h y_{t, h}-(n-h) E\left[y_{n-h, t+h} \mid y_{t}\right]}_{\text {spanned by } y_{t}}-\underbrace{(n-h)\left(E_{t}\left[y_{n-h, t+h}\right]-(n-h) E\left[y_{n-h, t+h} \mid y_{t}\right]\right)}_{\text {unspanned by } y_{t}} .
$$

So additional information will be useful for forecasting excess returns if it forecasts the component of future yields that is unspanned by $y_{t}$. With this in mind, we construct three forecast factors: (i) the yield forecast factor; (ii) the GDP forecast factor; and (iii) the inflation forecast factor. At each point in time, these factors are simply the average forecasts across all forecasters and all forecast horizons. The yield forecast factor is also an average across all yield maturities. Consistent with the construction of our surprise measures, forecasts for

the first two quarters are excluded from the averages. Assuming that market professionals 


\begin{tabular}{|c|c|c|c|c|c|c|c|c|c|}
\hline & \multirow{2}{*}{$\begin{array}{l}\mathrm{PC} 1-4 \\
\text { AdjR2 }\end{array}$} & \multicolumn{2}{|c|}{$\mathrm{PC} 1-4, \mathrm{~F}$} & \multirow{2}{*}{$\begin{array}{l}\mathrm{PC} 1-5 \\
\text { AdjR2 }\end{array}$} & \multicolumn{2}{|c|}{$\mathrm{PC} 1-5, \mathrm{~F}$} & \multirow{2}{*}{$\begin{array}{l}\text { PC1-6 } \\
\text { AdjR2 }\end{array}$} & \multicolumn{2}{|c|}{$\mathrm{PC} 1-6, \mathrm{~F}$} \\
\hline & & AdjR2 & pval & & $\operatorname{AdjR} 2$ & pval & & AdjR2 & pval \\
\hline \multicolumn{10}{|c|}{ Panel A: 3-month excess returns } \\
\hline Yield forcast factor & 0.088 & 0.091 & 0.298 & 0.121 & 0.122 & 0.377 & 0.156 & 0.153 & 0.943 \\
\hline GDP forecast factor & 0.088 & 0.085 & 0.870 & 0.121 & 0.118 & 0.878 & 0.156 & 0.154 & 0.764 \\
\hline Inflation forecast factor & 0.088 & 0.145 & 0.003 & 0.121 & 0.178 & 0.001 & 0.156 & 0.196 & 0.005 \\
\hline Mean jump amplitude & 0.088 & 0.151 & 0.004 & 0.121 & 0.194 & 0.001 & 0.156 & 0.225 & 0.001 \\
\hline \multicolumn{10}{|c|}{ Panel B: 12-month excess returns } \\
\hline Yield forcast factor & 0.367 & 0.375 & 0.145 & 0.382 & 0.388 & 0.181 & 0.431 & 0.430 & 0.753 \\
\hline GDP forecast factor & 0.367 & 0.370 & 0.636 & 0.382 & 0.384 & 0.651 & 0.431 & 0.436 & 0.533 \\
\hline Inflation forecast factor & 0.367 & 0.572 & 0.000 & 0.382 & 0.587 & 0.000 & 0.431 & 0.603 & 0.000 \\
\hline Mean jump amplitude & 0.367 & 0.555 & 0.000 & 0.382 & 0.583 & 0.000 & 0.431 & 0.625 & 0.000 \\
\hline
\end{tabular}

Table 8: Regressions of cross-maturity average bonds excess returns on the first $n$ yield PCs $(\mathrm{PC} 1-\mathrm{n})$ and the forecast factors $(\mathrm{F})$.

condition on more information than $y_{t}$, these forecast factors may be informative about the last term in (24). Additionally, we also consider the realized jump means variable (JM) constructed by Wright and Zhou (2009) and extended by Huang and Shi (2011). ${ }^{17}$ JM is a measure of the time-varying amplitude of jumps in the US Treasury bond market based on high frequency data on 30-year Treasury bond futures.

We proceed by projecting cross-maturity average excess returns on the first $n$ yield PCs for $n=4,5,6$, augmenting the projection with each of the four conditioning variables. The adjusted $R^{2}$ s are reported in Table 8 , where "pval" is the probability value for the t-statistic of the estimated coefficient on the included conditioning variable. Neither the yield-forecast factor nor the GDP-forecast factor shows any significant predictive power in the presence of yield PCs. In contrast, both the inflation-forecast factor and the JM variable show substantial predictive power for risk premiums, much stronger than the yield PCs. Moreover, the same results can be observed across different returns horizons, and independent of the number of PCs (up to six) included in the regressions. Therefore, simply increasing the number of yield-based factors is unlikely to overturn this result.

Given this evidence, and in the light of the strong link between $\varsigma_{t}^{2}$ and expected excess returns in ETSMs, the only way ETSMs may rationalize these results is through the presence of USV. In the next subsection, we take up the issue of USV and investigate whether USV within ETSMs can indeed rationalize the evidence documented here.

\section{USV in the Context of ETSMs}

What are the implications of the strong predictive power of the inflation factor (IF) and JM for excess returns for the specification of ETSMs? To develop the implications of

\footnotetext{
${ }^{17}$ We thank the authors of these papers for kindly providing us with both the original and the extended data series.
} 


\begin{tabular}{|c|c|c|c|c|c|c|c|c|c|}
\hline & \multicolumn{3}{|c|}{$H=2$} & \multicolumn{3}{|c|}{$H=4$} & \multicolumn{3}{|c|}{$H=6$} \\
\hline & \multicolumn{2}{|c|}{$\operatorname{AdjR} 2$} & \multirow[b]{2}{*}{ pval } & \multicolumn{2}{|c|}{$\operatorname{AdjR} 2$} & \multirow[b]{2}{*}{ pval } & \multicolumn{2}{|c|}{$\operatorname{AdjR} 2$} & \multirow[b]{2}{*}{ pval } \\
\hline & PC1-2 & IF & & PC1-2 & IF & & PC1-2 & IF & \\
\hline \multicolumn{10}{|c|}{ Panel A: Squared yield residuals } \\
\hline $3 \mathrm{~m}$ & 0.226 & 0.226 & 0.308 & 0.253 & 0.251 & 0.510 & 0.266 & 0.264 & 0.456 \\
\hline $5-\mathrm{y}$ & 0.220 & 0.227 & 0.238 & 0.266 & 0.264 & 0.662 & 0.262 & 0.261 & 0.394 \\
\hline $10-y$ & 0.273 & 0.284 & 0.064 & 0.318 & 0.318 & 0.229 & 0.314 & 0.316 & 0.138 \\
\hline \multicolumn{10}{|c|}{ Panel B: Macro residuals } \\
\hline$e_{C P I}^{2}$ & 0.286 & 0.309 & 0.118 & 0.338 & 0.350 & 0.244 & 0.369 & 0.379 & 0.261 \\
\hline$e_{G D P}^{2}$ & 0.322 & 0.320 & 0.086 & 0.392 & 0.392 & 0.406 & 0.433 & 0.431 & 0.299 \\
\hline$e_{C P I} e_{G D P}$ & -0.005 & -0.007 & 0.277 & 0.002 & 0.005 & 0.798 & 0.001 & -0.003 & 0.871 \\
\hline \multicolumn{10}{|c|}{ Panel C: Cross inflation-yield residual products } \\
\hline $3 \mathrm{~m}$ & 0.178 & 0.182 & 0.155 & $0.205^{*}$ & 0.207 & 0.314 & 0.243 & 0.241 & 0.369 \\
\hline $5-\mathrm{y}$ & 0.100 & 0.118 & 0.029 & 0.128 & 0.131 & 0.141 & 0.165 & 0.163 & 0.218 \\
\hline $10-\mathrm{y}$ & 0.109 & 0.124 & 0.068 & 0.135 & 0.137 & 0.349 & 0.164 & 0.162 & 0.533 \\
\hline \multicolumn{10}{|c|}{ Panel D: Cross GDP-yield residual products } \\
\hline $3 \mathrm{~m}$ & 0.062 & 0.059 & 0.676 & 0.077 & 0.073 & 0.210 & 0.095 & 0.095 & 0.142 \\
\hline $5-y$ & 0.032 & 0.029 & 0.842 & 0.042 & 0.039 & 0.879 & 0.046 & 0.045 & 0.909 \\
\hline $10-y$ & 0.042 & 0.038 & 0.830 & 0.050 & 0.047 & 0.733 & 0.057 & 0.055 & 0.829 \\
\hline
\end{tabular}

Table 9: Regressions of various squared residuals and residuals products constructed from BCFF yield forecasts on on the first two yield PCs and the inflation-forecast factor (IF).

unspanned risks within ETSMs, consider again the yield pricing equation (12). The presence of unspanned risks is equivalent to the rank of the loading matrix $[B, C]$ being less than the number of risk factors:

$$
r_{Z}=\operatorname{rank}([B, C])<N \text {. }
$$

At one extreme, suppose that $\varsigma_{t}^{2}$ is fully unspanned by bond yields. In this case the loadings $C$ are zero for all maturities, as $y_{t}$ cannot depend directly on $\varsigma_{t}^{2}$. For our empirical implementation where the total number of risk factors is $N=4$ and the number of volatility factors is $M=2$, this means that only the two non-volatility factors $x_{t}$ are spanned by $y_{t}$. Further, following the reasoning in Joslin, Le, and Singleton (2012), this implies that the first two yield PCs fully span the information generated by $x_{t}$.

More generally, when $r_{Z}<N$, it can be shown that there are $N-r_{Z}$ linear combinations of $\left(x_{t}^{\prime}, \varsigma_{t}^{2}\right)^{\prime}$ that cannot be inverted from bond yields. The information set generated by the $r_{Z}$ linear combinations of $\left(x_{t}^{\prime}, \varsigma_{t}^{2}\right)^{\prime}$ that can be inverted from bond yields should be well approximated by the first $r_{Z}$ yields PCs, particularly for small $r_{Z}$.

Now if $r_{Z}=1$, then yields at all maturities are perfectly correlated (conditionally and unconditionally). Accordingly, with $N=4$ in our empirical illustrations, realistic values of $r_{Z}$ are 2 and 3 . The larger is $r_{Z}$ the richer is the information set generated by the spanned components. To be conservative we set $r_{Z}=2$ in our subsequent analysis and use the first two yield PCs to control for the spanned components. This way, we give IF and JM the best 


\begin{tabular}{|c|c|c|c|c|c|c|c|c|c|}
\hline & \multicolumn{3}{|c|}{$H=2$} & \multicolumn{3}{|c|}{$H=4$} & \multicolumn{3}{|c|}{$H=6$} \\
\hline & \multicolumn{2}{|c|}{ AdjR2 } & \multirow[b]{2}{*}{ pval } & \multicolumn{2}{|c|}{$\operatorname{AdjR2}$} & \multirow[b]{2}{*}{ pval } & \multicolumn{2}{|c|}{ AdjR2 } & \multirow[b]{2}{*}{ pval } \\
\hline & PC1-2 & $\mathrm{JM}$ & & PC1-2 & $\mathrm{JM}$ & & PC1-2 & $\mathrm{JM}$ & \\
\hline \multicolumn{10}{|c|}{ Panel A: Squared yield residuals } \\
\hline $3 \mathrm{~m}$ & 0.226 & 0.237 & 0.223 & 0.253 & 0.268 & 0.068 & 0.266 & 0.282 & 0.086 \\
\hline $5-\mathrm{y}$ & 0.220 & 0.217 & 0.401 & 0.266 & 0.266 & 0.133 & 0.262 & 0.262 & 0.127 \\
\hline $10-y$ & 0.273 & 0.273 & 0.501 & 0.318 & 0.316 & 0.774 & 0.314 & 0.312 & 0.967 \\
\hline \multicolumn{10}{|c|}{ Panel B: Macro residuals } \\
\hline$e_{C P I}^{2}$ & 0.286 & 0.306 & 0.784 & 0.338 & 0.345 & 0.845 & 0.369 & 0.371 & 0.707 \\
\hline$e_{G D P}^{2}$ & 0.322 & 0.321 & 0.746 & 0.392 & 0.389 & 0.980 & 0.433 & 0.432 & 0.968 \\
\hline$e_{C P I} e_{G D P}$ & -0.005 & -0.009 & 0.643 & 0.002 & -0.001 & 0.543 & 0.001 & 0.000 & 0.499 \\
\hline \multicolumn{10}{|c|}{ Panel C: Cross inflation-yield residual products } \\
\hline $3 \mathrm{~m}$ & 0.178 & 0.176 & 0.846 & 0.205 & 0.202 & 0.798 & 0.243 & 0.240 & 0.286 \\
\hline $5-\mathrm{y}$ & 0.100 & 0.112 & 0.37 & 0.128 & 0.133 & 0.729 & 0.165 & 0.168 & 0.877 \\
\hline $10-y$ & 0.109 & 0.123 & 0.249 & 0.135 & 0.143 & 0.429 & 0.164 & 0.170 & 0.502 \\
\hline \multicolumn{10}{|c|}{ Panel D: Cross GDP-yield residual products } \\
\hline $3 \mathrm{~m}$ & 0.062 & 0.062 & 0.999 & 0.077 & 0.078 & 0.817 & 0.095 & 0.100 & 0.637 \\
\hline $5-y$ & 0.032 & 0.035 & 0.35 & 0.042 & 0.045 & 0.540 & 0.046 & 0.056 & 0.649 \\
\hline $10-y$ & 0.042 & 0.042 & 0.330 & 0.050 & 0.051 & 0.438 & 0.057 & 0.064 & 0.739 \\
\hline
\end{tabular}

Table 10: Regressions of various squared residuals and residuals products constructed from $\mathrm{BCFF}$ yield forecasts on on the first two yield PCs and the jump means variable.

chances to exhibit their predictive power for squared residuals. For robustness, we also repeat the same analysis with $r_{Z}=3$ (and $r_{Z}=4$ to account for models with $N>4$ ) and obtain very comparable results (not reported).

We report the projections of yield squared residuals onto the first two PCs and the IF (JM) variable in Panel A of Table 9 (Table 10). For brevity, we only include three maturities (3 months, 5 years, and 10 years) as the results for other maturities are very similar. Interestingly, despite their power for excess returns, neither the IF nor the JM variable displays any significant incremental predictive power for yield squared residuals. The adjusted $R^{2}$ s by adding the conditioning variables only increase modestly in most cases. All of the probability values ("pval") for the t-statistics associated with the estimated coefficients of the conditioning variables are larger than $5 \%$, and most of them larger than $10 \%$. Placing this evidence in the context of ETSMs, it suggests that the predictive power of both the IF and the JM variable does not seem to originate from unspanned yield volatility. We emphasize that this should not be interpreted as evidence against the existence of unspanned volatility, but rather that unspanned volatility of yields is unlikely behind the predictive power of neither the IF nor the JM variable for bonds excess returns.

To see how such a scenario might be plausible, imagine a scenario that corresponds to our empirical setup $\left(N=4, M=2, r_{Z}=2\right)$ and assume that one of the non-volatility factors $\left(x_{1, t}\right)$ and one of the volatility factors $\left(\varsigma_{1, t}^{2}\right)$ are unspanned (that is, their respective loadings on the yield pricing equations are uniformly zeros) and the remaining factors $\left(x_{2, t}, \varsigma_{2, t}^{2}\right)$ fully 
spanned. Furthermore, assume that the spanned volatility, $\varsigma_{2, t}^{2}$, sufficiently modulates the time varying volatility of both spanned variables $\left(x_{2, t}, \varsigma_{2, t}^{2}\right)$, but both volatility factors are needed to fully capture the predictability of bonds excess returns. In such a setting, if the IF factor and the JM variable are informative about $\varsigma_{1, t}^{2}$, they would be able to forecast excess returns beyond the yields PCs without showing any incremental power in predicting yield squared residuals.

Pursuing the above example, noting that the conditional variance of any variable within the ETSMs must be driven by a linear combination of $\varsigma_{1, t}^{2}$ and $\varsigma_{2, t}^{2}$, any economic variables whose variances are sufficiently distinct from yields volatility must be relatively informative about the unspanned volatility factor $\varsigma_{1, t}^{2}$, and hence the source of predictive power of the IF factor and the JM variable. Guided by this intuition, we next investigate the possibility of unspanned inflation volatility and unspanned GDP volatility as potential sources for the predictive power of the two conditioning variables being considered. Similar analysis is conducted using inflation and GDP squared residuals as well as the product of inflation and GDP residuals constructed from earlier sections. The results are reported in Panel B of Table 9 and Table 10. Very similar to the results for yields volatility, both the IF and JM variables show mostly insignificant ability to predict the squared residuals (and the product of residuals) beyond the first two yield PCs. It is evident that neither unspanned inflation volatility nor unspanned GDP volatility is likely responsible for the predictability documented earlier in this section.

Finally, following an observation from the preceding section that within ETSMs, the conditional covariances between yields and inflation as well as the conditional covariances between yields and real GDP must also be linear in $\varsigma_{t}^{2}$, we perform similar projections of the cross-products of inflation (GDP) and yield forecast residuals onto PC1-2 and the two conditioning variables being analysed. The results are reported in Panel $\mathrm{C}$ and Panel $\mathrm{C}$ of Table 9 and Table 10. Again, both the IF and JM variables fail to show any predictive power for these residuals products above the yield PCs.

Taken together, these findings cast doubt on the presumption of many ETSMs that variation in expected excess returns is induced entirely by time varying volatility of the state variables. The predictive power of an inflation factor is consistent with evidence reported in Joslin, Priebsch, and Singleton (2011) and Cieslak and Povala (2011). Our analysis goes further by showing that the impact of expected inflation on risk premiums is not operating through its affect on time-varying quantities of risk in the US Treasury bond market. A likely alternative channel is through its affects on the market prices of risk, and the latter are constant or nearly constant (at estimated parameter values) in most extant ETSMs.

\section{Concluding Remarks}

In this paper, we set out to explore in depth the nature of risk premiums in US Treasury bond markets over the past thirty years through the lens of investors' pricing kernels as parameterized in studies of preference-based ETSMs. Many prominent equilibrium term structure models $(E T S M \mathrm{~s})$ in which the state of the economy $z_{t}$ follows an affine process 
imply that the variation in expected excess returns on bond portfolio positions is fully spanned by the set of conditional variances $\varsigma_{t}^{2}$ of $z_{t}$. We show that these two assumptions alone- spanning of excess returns by the variances $\varsigma_{t}^{2}$ of affine processes $z_{t}-$ are sufficient to econometrically identify the quantities of risk that span risk premiums from the term structure of bond yields. Using this result we derive maximum likelihood estimates of $\varsigma_{t}^{2}$ and evaluate the goodness-of-fit of the family of affine ETSMs that imply this tight link between premiums and quantities of risk. These assessments are fully robust to the values of the parameters governing preferences and the evolution of the state $z_{t}$, and to whether or not the economy is arbitrage free. Our findings suggest that, to be consistent with U.S. macroeconomic and Treasury yield data, affine ETSMs should have the features that: (i) the fundamental sources of risks, including consumption growth, inflation, and yield volatilities are driven by distinct economic shocks; (ii) consumption growth risk alone is unlikely to fully account for the predictability of excess returns on bonds; and (iii) inflation risk, and not long-run risks or variation in risk premiums arising from habit-based preferences, is likely to be the dominant risk underlying risk premiums in U.S. Treasury markets. 


\section{A Construction of Zero Yield Forecasts}

In this section, we give details of the construction of the zero yield forecasts used in the paper. We first show how to interpolate the raw forecasts (of yields over calendar quarters) obtain forecasts for non-calendar quarters. Next, we show how to construct zero yield forecasts from forecasts of par yields. To fix notation, let's denote the n-period zero yields and n-period par yields by $y_{n, t}$ and $\tilde{y}_{n, t}$, respectively.

\section{A.1 Non-Calendar Quarter Forecasts}

Recall that from the Blue Chip surveys, we obtain forecasts of average par yields over calendar quarters. For example, the one- and two-quarter forecasts as of the end of December, January, and February are all:

$$
\tilde{F}_{1, t}=E_{t}\left[\tilde{y}_{120, J a n}+\tilde{y}_{120, F e b}+\tilde{y}_{120, M a r}\right] \text { and } \tilde{F}_{2, t}=E_{t}\left[\tilde{y}_{120, A p r}+\tilde{y}_{120, M a y}+\tilde{y}_{120, J u n}\right] .
$$

Obviously, the forecast horizons are different from one month to another depending on which month of the quarter at which the forecasts are formed. To equate the forecast horizons throughout the sample, we follow the approach of Chun (2010) and interpolate the raw forecasts such that the one-quarter forecasts are always for the average par yields of the first three months from $t+1$ to $t+3$, the two-quarter forecasts are for the average par yields from $t+4$ to $t+6:$

$$
\tilde{Q}_{1, t}=E_{t}\left[\tilde{y}_{120, t+1}+\tilde{y}_{120, t+2}+\tilde{y}_{120, t+3}\right], \quad \tilde{Q}_{2, t}=E_{t}\left[\tilde{y}_{120, t+4}+\tilde{y}_{120, t+5}+\tilde{y}_{120, t+6}\right],
$$

and so on. Specifically, for the first month of each quarter (Jan, Apr, Jul, Oct), we compute the q-quarter forecasts as:

$$
\tilde{Q}_{q, t}=\frac{2}{3} \tilde{F}_{q, t}+\frac{1}{3} \tilde{F}_{q+1, t}
$$

Likewise, for the second month of each quarter (Feb, May, Aug, Nov), we compute the q-quarter forecasts as:

$$
\tilde{Q}_{q, t}=\frac{1}{3} \tilde{F}_{q, t}+\frac{2}{3} \tilde{F}_{q+1, t}
$$

For the third month of each quarter, we leave the raw forecasts intact. The same treatment is applied to bonds of all maturities.

\section{A.2 Zero Yield Forecasts}

For each day $t$ and each forecast horizon $q$, we have forecasts for par yields of various maturities. The set of maturities has changed from time to time. For the first four years of the sample (1984-1987), there are only three maturities included: 3-month, 3-year, and 30-year. For the remaining twenty years of the sample, forecasts of eight different maturities are reported each 
month. Except for some brief changes, ${ }^{18}$ this set includes: 3-month, 6-month, 1-year, 2-year, 5-year, 7-year, 10-year, and 30-year.

To fix to a set of maturities over time, and more importantly, to convert forecasts of par yields into forecasts of corresponding zero yields, we simply apply the standard Fama-Bliss bootstrap technique ${ }^{19}$ to the set of par yield forecasts on each day $t$ and each forecast horizon $q$. In so doing, we treat the par yield forecasts as if they were actual observed par yields. Additionally, in the bootstrapping calculations, we ignore the fact that the forecasts are for averages over three successive months. The output of the bootstrap, for example for the 10-year maturity and one-quarter forecast horizon, is interpreted as:

$$
Q_{1, t}=E_{t}\left[y_{120, t+1}+y_{120, t+2}+y_{120, t+3}\right] .
$$

In applying the bootstrapping techniques to the average forecasts directly, ${ }^{20}$ the resulting zero yield forecasts suffer from the Jensen effect. However, we now show that this effect is economically very negligible.

To prove that the Jensen effect is very minimal, we perform the following exercise. We fit yields data over our sample period to three prominent term structure models: $A_{0}(4), F_{1}(4)$, and $F_{2}(4)$. The first model is the standard gaussian affine with no-arbitrage. The next two models have one and two stochastic volatility factors, respectively but without no-arbitrage imposed. To be consistent with the models considered in this paper, we choose four factors in each model. Next, we use each model to generate par yield forecasts for six different maturities, ${ }^{21}$ averaged over three-month windows, corresponding exactly to the forecasts reported in the BCFF newsletters. To each set of forecasts, we apply the bootstrapping technique described above to obtain an approximate set of zero yield forecasts averaged over three-month windows. Finally, we compare these bootstrapped forecasts to the true forecasts of zero yields generated by each model.

Figure 3 plots the bootstrapped forecasts and the true forecasts generated by the $F_{2}(4)$ model for the 1-year and 10-year bonds:

$$
E_{t}\left[y_{12, t+16}+y_{12, t+17}+y_{12, t+18}\right] \text { and } E_{t}\left[y_{120, t+16}+y_{120, t+17}+y_{120, t+18}\right] \text {. }
$$

Note that these forecasts are for six quarters out. Visually, the bootstrapped and the true forecasts are essentially identical. Very similar pattern obtains if we use the other two models, any forecast horizon or any bond maturity.

To show this more concretely, we regress the approximate forecasts on the true forecasts and report the loading, the adjusted R-squared statistics in Table 11. Evidently, regardless

\footnotetext{
${ }^{18}$ For example, the 30 -year series were replaced briefly by the 20 -year series when the former was discontinued and resumed again.

${ }^{19}$ Specifically, we assume that the forward rates are piece-wise constant between any two successive maturities.

${ }^{20}$ The more proper approach is to apply the bootstrapping technique to each future realization of the par yield curve and take average over all resulting zero curves. But such a method is not feasible since we do not observe the dynamics underlying each forecast.

${ }^{21}$ They are 6-month, 1-year, 3-year, 5-year, 7-year, and 10-year.
} 


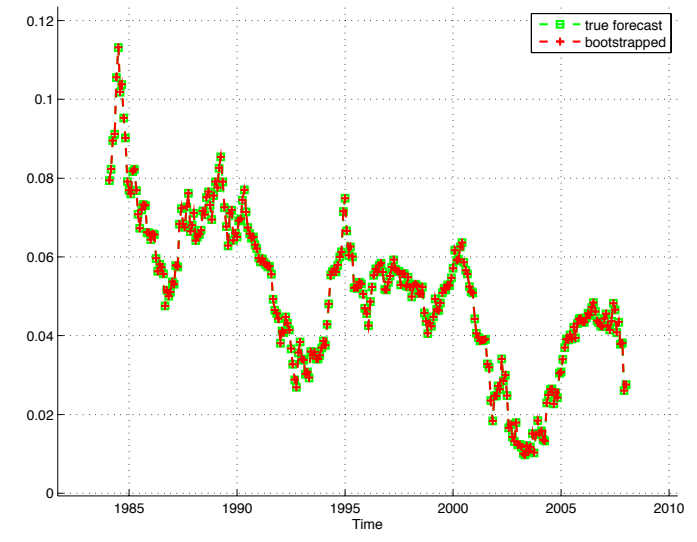

(a) 1 -yr bond, $F_{2}(4)$

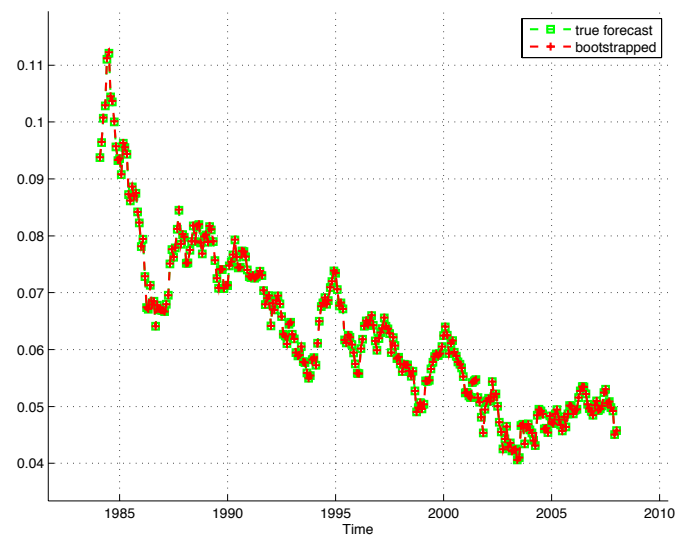

(b) 10-year bond, $F_{2}(4)$

Figure 3: Comparison of bootstrapped forecasts versus true forecasts of average zero-yields averaged over the sixth quarter.

of the forecast horizons (three quarters or six quarters out), bond maturity (1-year, 3-year, 5-year, or 10-year), or the true underlying models $\left(A_{0}(4), F_{1}(4)\right.$, or $\left.F_{2}(4)\right)$, the adjusted R-squared is always perfect and the loading is essentially one. The average differences as well as the RMSEs between the bootstrapped and true forecasts are, for all cases, less than or equal to three basis points.

\section{B $A R G(\rho, c, \nu)$ Processes}

Following Le, Singleton, and Dai (2010) we assume that, conditional on $\varsigma_{t}^{2}$, the components of $\varsigma_{t+1}^{2}$ are independent. To specify the conditional distribution of $\varsigma_{t+1}^{2}$, we let $\varrho$ be an $N \times N$ matrix with elements satisfying

$$
0<\varrho_{i i}<1, \varrho_{i j} \leq 0,1 \leq i, j \leq N .
$$

Furthermore, for each $1 \leq i \leq N$, we let $\rho_{i}$ be the $i^{\text {th }}$ row of the $N \times N$ non-singular matrix $\rho=\left(I_{N \times N}-\varrho\right)$. Then, for constants $c_{i}>0, \nu_{i}>0, i=1, \ldots, N$, we define the conditional density of $\varsigma_{i, t+1}^{2}$ given $\varsigma_{t}^{2}$ as the Poisson mixture of standard gamma distributions:

$$
\frac{\varsigma_{i, t+1}^{2}}{c_{i}} \mid\left(P, \varsigma_{t}^{2}\right) \sim \operatorname{gamma}\left(\nu_{i}+P\right), \text { where } P \mid \varsigma_{t}^{2} \sim \operatorname{Poisson}\left(\rho_{i} \varsigma_{t}^{2} / c_{i}\right) .
$$

Here, the random variable $P \in(0,1,2, \ldots)$ is drawn from a Poisson distribution with intensity modulated by the current realization of the state vector $\varsigma_{t}^{2}$. 


\begin{tabular}{|c|c|c|c|c|c|c|c|c|c|}
\hline & & \multicolumn{4}{|c|}{ Three-quarter forecasts } & \multicolumn{4}{|c|}{ Six-quarter forecasts } \\
\hline & & Loading & Adj R2 & Ave. Diff. & RMSE & Loading & Adj R2 & Ave. Diff. & RMSE \\
\hline \multirow{4}{*}{$A_{0}(4)$} & 1 -yr bond & 1.000 & 1.000 & -2.436 & 2.436 & 1.000 & 1.000 & -0.999 & 0.999 \\
\hline & 3-yr bond & 1.000 & 1.000 & -2.446 & 2.463 & 1.000 & 1.000 & -0.251 & 0.317 \\
\hline & 5 -yr bond & 1.000 & 1.000 & -2.426 & 2.439 & 1.000 & 1.000 & -0.206 & 0.268 \\
\hline & 10-yr bond & 1.001 & 1.000 & -2.388 & 2.401 & 1.001 & 1.000 & -0.576 & 0.608 \\
\hline \multirow{4}{*}{$F_{1}(4)$} & 1-yr bond & 0.999 & 1.000 & 2.018 & 2.027 & 1.001 & 1.000 & -0.752 & 0.799 \\
\hline & 3-yr bond & 1.000 & 1.000 & 2.931 & 2.943 & 1.001 & 1.000 & -0.380 & 0.417 \\
\hline & 5 -yr bond & 1.000 & 1.000 & 3.034 & 3.042 & 1.001 & 1.000 & -0.584 & 0.624 \\
\hline & 10-yr bond & 0.999 & 1.000 & 2.778 & 2.796 & 1.001 & 1.000 & -1.119 & 1.144 \\
\hline \multirow{4}{*}{$F_{2}(4)$} & 1-yr bond & 1.000 & 1.000 & -0.425 & 0.446 & 1.000 & 1.000 & -0.274 & 0.292 \\
\hline & $3-y r$ bond & 1.001 & 1.000 & -0.515 & 0.559 & 1.001 & 1.000 & 0.274 & 0.327 \\
\hline & 5-yr bond & 1.001 & 1.000 & -0.812 & 0.866 & 1.001 & 1.000 & 0.174 & 0.299 \\
\hline & 10 -yr bond & 1.002 & 1.000 & -1.175 & 1.250 & 1.002 & 1.000 & -0.227 & 0.408 \\
\hline
\end{tabular}

Table 11: Regression of bootstrapped forecasts on true forecasts. Average differences (Ave. Diff.) and root mean squared errors (RMSE) are in basis points.

The conditional density function of $\varsigma_{i, t+1}^{2}$ takes the form:

$$
f^{\mathbb{Q}}\left(\varsigma_{i, t+1}^{2} \mid \varsigma_{t}^{2}\right)=\frac{1}{c_{i}} \sum_{k=0}^{\infty}\left[\frac{\left(\frac{\rho_{i} \varsigma_{t}^{2}}{c_{i}}\right)^{k}}{k !} e^{-\frac{\rho_{i} \varsigma_{t}^{2}}{c_{i}}} \times \frac{\left(\frac{\varsigma_{i, t+1}^{2}}{c_{i}}\right)^{\nu_{i}+k-1} e^{-\frac{\varsigma_{i, t+1}^{2}}{c_{i}}}}{\Gamma\left(\nu_{i}+k\right)}\right] .
$$

Using conditional independence, the distribution of $\varsigma_{t+1}^{2}$, conditional on $\varsigma_{t}^{2}$, is given by $f\left(\varsigma_{t+1}^{2} \mid \varsigma_{t}^{2}\right)=\prod_{i=1}^{N} f\left(\varsigma_{i, t+1}^{2} \mid \varsigma_{t}^{2}\right)$. When the off-diagonal elements of the $N \times N$ matrix $\varrho$ are non-zero, the autoregressive gamma processes $\left\{\varsigma_{i}^{2}\right\}$ are (unconditionally) correlated. Thus, even in the case of correlated $\varsigma_{i t}^{2}$, the conditional density of $\varsigma_{i, t+1}^{2}$ is known in closed form. 


\section{References}

Bansal, R., D. Kiku, and A. Yaron (2007). Risks for the long run: Estimation and inference. Technical report, Duke University.

Bansal, R. and I. Shaliastovich (2010). Risk and return in bond, currency and equity markets. Technical report, Duke University.

Bansal, R. and A. Yaron (2004). Risks for the long run: A potential resolution of asset pricing puzzles. Journal of Finance 59, 1481-1509.

Beeler, J. and J. Campbell (2009). The long-run risks model and aggregate asset prices: An empirical assessment. Technical report, Harvard University.

Bekaert, G. and E. Engstrom (2010). Asset return dynamics under band environment-good environment fundamentals. Technical report, Columbia University.

Bliss, R. R. (1997). Testing term structure estimation methods. Advances in Futures and Options Research 9, 197-231.

Bollerslev, T., G. Tauchen, and H. Zhou (2009). Expected stock returns and variance risk premia. Review of Financial Studies 22, 4463-4492.

Bonomo, M., R. Garcia, N. Meddahi, and R. Tedongap (2010). Disappointment aversion, longrun risks and aggregate asset prices. Technical report, forthcoming, Review of Financial Studies.

Campbell, J. and J. Cochrane (1999). By force of habit: A consumption-based explanation of aggregate stock market behavior. Journal of Political Economy 107, 205-251.

Chun, A. (2010). Expectations, bond yields and monetary policy. Technical report, forthcoming, Review of Financial Studies.

Cieslak, A. and P. Povala (2011). Understanding bond risk premia. Technical report, University of Lugano.

Cochrane, J. and M. Piazzesi (2005). Bond risk premia. American Economic Review 95, $138-160$.

Collin-Dufresne, P. and R. S. Goldstein (2002). Do bonds span the fixed income markets? theory and evidence for 'unspanned' stochastic volatility. Journal of Finance 5\%, 1685-1730.

Constantinides, G. and A. Ghosh (2009). Asset pricing tests with long run risks in consumption growth. Technical report, Graduate School of Business, University of Chicago.

Cox, J., J. Ingersoll, and S. Ross (1985). An intertemporal general equilibrium model of asset prices. Econometrica 53, 363-384. 
Dai, Q. and K. Singleton (2000). Specification analysis of affine term structure models. Journal of Finance 55, 1943-1978.

Dai, Q. and K. Singleton (2003). Term structure dynamics in theory and reality. Review of Financial Studies 16, 631-678.

Doh, T. (2011). Long run risks in the term structure of interest rates: Estimation. Technical report, Federal Reserve Bank of Kansas City, RWP 08-11.

Drechsler, I. and A. Yaron (2011). What's vol got to do with it. Review of Financial Studies 24.

Duarte, J. (2008). The causal effect of mortgage refinancing on interest-rate volatility: Empirical evidence and theoretical implications. Review of Financial Studies 21, 16891731.

Duffee, G. (2011). Information in (and not in) the term structure. Review of Financial Studies 24, 2895-2934.

Duffie, D., J. Pan, and K. Singleton (2000). Transform analysis and asset pricing for affine jump-diffusions. Econometrica 68, 1343-1376.

Engle, R. (1982). Autoregressive conditional heteroskedasticity with estimates of the variance of u.k. inflation. Econometrica 50, 987-1008.

Eraker, B. (2008). Affine general equilibrium models. Management Science 54, 2068-2080.

Eraker, B. and I. Shaliastovich (2008). An equilibrium guide to designing affine asset pricing models. Mathematical Finance 18, 519-543.

Fama, E. F. and R. R. Bliss (1987). The information in long-maturity forward rates. American Economic Review r7(4), 680-692.

Gallmeyer, M., B. Hollifield, and S. Zin (2005). Taylor rules, mccallum rules and the term structure of interest rates. Journal of Monetary Economics 52, 921-950.

Gourieroux, C. and J. Jasiak (2006). Autoregressive gamma processes. Journal of Forecasting 25, 129-152.

Greenwood, R. and D. Vayanos (2010a). Bond supply and excess bond returns. Technical report, LSE.

Greenwood, R. and D. Vayanos (2010b). Price pressure in the government bond market. American Economic Review P\&P, 585-590.

Gurkanyak, R., B. Sack, and J. Wright (2007). The U.S. treasury yield curve: 1961 to the present. Journal of Monetary Economics 54, 2291-2304. 
Hansen, L., J. Heaton, and N. Li (2008). Consumption strikes back? measuring long-run risk. Journal of Political Economy 116, 260-302.

Huang, J. and Z. Shi (2011). Determinants of bond risk premia. Technical report, Penn State University.

Joslin, S. (2011). Can unspanned stochastic volatility models explain the cross section of bond volatilities? Technical report, USC.

Joslin, S., A. Le, and K. Singleton (2012). Why gaussian macro-finance term structure models are (nearly) unconstrained factor-vars. Journal of Financial Economics, forthcoming.

Joslin, S., M. Priebsch, and K. Singleton (2011). Risk premiums in dynamic term structure models with unspanned macro risks. Technical report, Stanford University.

Joslin, S., K. Singleton, and H. Zhu (2011). A new perspective on gaussian dynamic term structure models. Review of Financial Studies 24, 926-970.

Koijen, R., H. Lustig, S. Van Nieuwerburgh, and A. Verdelhan (2010). Long-run risk, the wealth consumption ratio, and the temporal pricing of risk. American Economic Review 100, $552-556$.

Krishnamurthy, A. and A. Vissing-Jorgensen (2010). The aggregate demand for treasury debt. Technical report, Kellogg School, Northwestern University.

Le, A., K. Singleton, and J. Dai (2010). Discrete-time affine ${ }^{q}$ term structure models with generalized market prices of risk. Review of Financial Studies 23, 2184-2227.

Li, H. and F. Zhao (2006). Unspanned stochastic volatility: Evidence from hedging interest rate derivatives. Journal of Finance 61, 341-378.

Litterman, R., J. Scheinkman, and L. Weiss (1991). Volatility and the yield curve. Journal of Fixed Income 1, 49-53.

Longstaff, F. A. and E. S. Schwartz (1992). Interest rate volatility and the term structure: A two-factor general equilibrium model. Journal of Finance 47, 1259-1282.

Ludvigson, S. and S. Ng (2010). Macro factors in bond risk premia. Review of Financial Studies 22, 5027-5067.

Marakani, S. (2009). Long run consumption risks: Are they there? Technical report, Northwestern University.

McCallum, B. T. (1994). Monetary policy and the term structure of interest rates. NBER Working Paper No. 4938.

Nelson, C. and A. Siegel (1987). Parsimonious modelling of yield curves. Journal of Business 60, 473-489. 
Piazzesi, M. (2010). Affine term structure models. In Y. Ait-Sahalia and L. Hansen (Eds.), Handbook of Financial Econometrics, Chapter 12, pp. 691-766. Elsevier B.V.

Piazzesi, M. and M. Schneider (2007). Equilibrium yield curves. In D. Acemoglu, K. Rogoff, and M. Woodford (Eds.), NBER Macroeconomics Annual. Cambridge, MA: MIT Press.

Rudebusch, G. and T. Wu (2007). Accounting for a shift in term structure behavior with no-arbitrage and macro-finance models. Journal of Money Credit and Banking 39, 395 422.

Schwarz, G. (1978). Estimating the dimension of a model. Annals of Statistics 6, 461-464.

Stock, J. and M. Watson (2002). Has the business cycle changed and why? Technical report, NBER Macroeconomics Annual.

Stock, J. and M. Watson (2007). Why has u.s. inflation become harder to forecast? Journal of Money Credit and Banking 39, 3-33.

Vayanos, D. and J. Vila (2009). A preferred-habitat model of the term structure of interest rates. Technical report, London School of Economics.

Wachter, J. A. (2006). A consumption-based model of the term structure of interest rates. Journal of Financial Economics 79(2), 365-399.

Wright, J. (2011). Term premiums and inflation uncertainty: Empirical evidence from an international panel dataset. American Economic Review 101, 1514-34.

Wright, J. and H. Zhou (2009). Bond risk premia and realized jump risk. Journal of Banking and Finance 33, 2333-2345. 